\title{
A comparative study of conditional moment closure modelling for ignition of iso-octane and $n$-heptane in thermally stratified mixtures
}

\author{
Fatemeh Salehi · Mohsen Talei • \\ Evatt R. Hawkes • Chun Sang Yoo • \\ Tommaso Lucchini • Gianluca D'Errico • \\ Sanghoon Kook
}

Received: date / Accepted: date

\begin{abstract}
This paper presents a comparative study of the premixed conditional moment closure (CMC) model for modelling ignition of thermally
\end{abstract}

Fatemeh Salehi

School of Photovoltaic and Renewable Energy Engineering,

The University of New South Wales, NSW 2052, Australia

Tel.: +61-2-9385-6196

Fax: +61-2-9385-7762

E-mail: f.salehi@unsw.edu.au

Mohsen Talei

Department of Mechanical Engineering,

University of Melbourne, Parkville, VIC 3010, Australia

Evatt R. Hawkes

School of Photovoltaic and Renewable Energy Engineering,

School of Mechanical and Manufacturing Engineering,

The University of New South Wales, NSW 2052, Australia

Chun Sang Yoo

School of Mechanical and Nuclear Engineering,

Ulsan National Institute of Science and Technology, Ulsan 689-798, Republic of Korea

Tommaso Lucchini

Department of Energy,

Politecnico di Milano, Milan, 20156, Italy

Gianluca D'Errico

Department of Energy,

Politecnico di Milano, Milan, 20156, Italy

Sanghoon Kook

School of Mechanical and Manufacturing Engineering,

The University of New South Wales, NSW 2052, Australia 
stratified mixtures under homogeneous charge compression ignition (HCCI) conditions. For this purpose, the CMC model is applied to two sets of direct numerical simulations (DNSs) modelling ignition of lean $n$-heptane/air and iso-octane/air mixtures with various levels of thermal stratification. The results show excellent agreement for all $n$-heptane cases with thermal stratification of $15-60 \mathrm{~K}$. However, an advanced ignition is predicted by the CMC model for the iso-octane case with thermal stratification of $60 \mathrm{~K}$ in comparison with the DNS data. Inspection of homogeneous ignition delay demonstrates that the ignition delay time fluctuations are much higher in the iso-octane cases compared with the $n$-heptane cases having same level of temperature inhomogeneities. This is because of the differing ignition responses to temperature between these two fuels. The observed discrepancies in the iso-octane case with $T^{\prime}=60 \mathrm{~K}$ are due to the dominance of deflagration mode of combustion resulting in large conditional fluctuations, which occurs in the iso-octane case and not the $n$-heptane case because the temperature dependence of ignition delay is stronger for iso-octane. To further investigate the reasons for the observed discrepancies, a transport equation for the conditional variance is derived for premixed combustion. Assessment of the conditional variance equation using the DNS data shows that correlations between dissipation and conditional fluctuation and correlations between reaction and conditional fluctuations are the dominant sources of conditional fluctuations.

Keywords Conditional moment closure - thermal stratification - HCCI · $n$-heptane $\cdot$ iso-octane

\section{Introduction}

Homogeneous charge compression ignition ( $\mathrm{HCCI}$ ) is a fairly recent engine technology developed to reduce emissions and improve combustion efficiency. In an HCCI engine, a lean or highly diluted and nearly homogeneous mixture of air and fuel (and possibly some combustion products) is used to achieve extremely low levels of $\mathrm{NO}_{\mathrm{x}}$ and soot. The fuel efficiency is also very high, similar to a diesel engine, due mainly to the fact that throttling is avoided since load control is achieved by altering the fuelling rate, but also due to the fact that peak combustion temperatures are low, resulting in lower heat transfer and dissociation losses. In spite of these favourable features of HCCI combustion, it presents considerable challenges such as difficulties in controlling the autoignition timing, especially during transients, and the high pressure rise at high load [1]. As a result, HCCI is still in the phase of laboratory research and has not seen commercial application except in demonstrations, for example in a demonstration which used HCCI only at part load [2].

As widely reported in the literature, thermal stratification of the charge can spread out the heat release rate profile and therefore reduce the pressure rise rate $[1,3,4,5,6]$. Thermal stratification naturally occurs in all HCCI engines [1, $3,4,5,6,7]$ due to various reasons such as inhalation of the charge with a nonuniform temperature distribution, wall heat transfer and incomplete mixing 
between the fresh charge and hot residuals or between fuel and air in the case of early direct injection or port injection $[1,3]$. Thermal stratification leads to sequential autoignition over a range of different times and in different regions inside the cylinder and therefore reduces the pressure-rise and heat release rates $[3,5]$.

The effect of thermal stratification under HCCI conditions has been investigated using direct numerical simulation (DNS) for various fuels such as hydrogen $[8,9,10,11], n$-heptane $[12,13]$ iso-octane [14] and dimethyl ether $[15,16]$. From some of these DNS studies, two combustion modes were found under HCCI conditions: deflagration and spontaneous ignition. In the deflagration mode, importance of molecular transport (diffusion and convection) becomes significant in comparison with reaction whereas the spontaneous mode presents simultaneous ignition events in which the role of molecular transport is negligible and different regions are coupled only by pressure [8,9]. The DNS data demonstrated that the deflagration combustion mode was predominant in cases with large thermal stratifications which the mean heat release rate was spread out. On the other hand, the spontaneous combustion mode was found to prevail in cases with a low level of temperature inhomogeneities which featured a high pressure-rise rate $[8,9,13,15]$. The DNS studies have also showed that increasing the level of thermal stratification generally advanced the ignition. However, for fuels such as $n$-heptane [13,12] and dimethyl ether [15] which exhibit negative temperature coefficient (NTC) behaviour, depending on the mean temperature a higher stratification level can retard the ignition. Although DNS is able to provide a detailed understanding of ignition characteristics under HCCI conditions, it can not be used as a design tool to simulate the combustion process in HCCI engines. Therefore, a number of numerical models which are computationally more affordable than the DNS for engine simulations have been proposed.

One common approach to model combustion under HCCI conditions is the multi-zone model $[17,18]$ in which a number of homogeneous zones are employed to represent the in-cylinder conditions [17]. These zones are only coupled by pressure and mixing between zones is not considered. Therefore, this model is only applicable in the spontaneous ignition regime, and a poor performance has been noted when the level of inhomogeneity and the importance of mixing increase [19].

HCCI-like conditions have been also modelled using flamelet-based approaches. A combined model based on a flamelet approach was developed in Ref. [20] in which the reaction rates were calculated based on the solution of a steady-state flamelet equation. Pitsch and co-workers [19,21] developed an enthalpy-based flamelet model in which the transport equations in the physical domain were transformed on a system of coordinates constructed based on enthalpy gradients. This new system includes a normal unit vector to the enthalpy iso-surface and two tangential unit vectors. It was assumed that the diffusion terms in the direction tangential to the enthalpy iso-surface were negligible. In a separate study, they extended the enthalpy-based flamelet model to a two-dimensional mixture-fraction and enthalpy based flamelet model to 
study effects of thermal and mixture stratification under HCCI conditions [22]. A good agreement with the experimental pressure trace was observed while unburned hydrocarbon emissions were over predicted.

The G-equation model combined with a multi-zone approach was applied to spark-assisted HCCI engines [23]. In this model, an extended form of the G-equation model was used for tracking the flame propagation whereas autoignition was captured by a multi-zone model. It was shown that this mixedmode combustion model is required to capture the correct behaviour under HCCI conditions.

Jay and co-workers [24] developed a method based on tabulated reaction rates to simulate HCCI-like configurations. The prediction of the model to capture the early heat release was found to be accurate however the model over-predicted the peak pressure in comparison with the experiment. In a separate study, they applied a more advanced tabulation model based on intrinsic low dimensional manifold (ILDM) and flamelet models [25]. The performance of the model for homogeneous, constant volume reactors and diesel-engine conditions was found satisfactory. However, the model had a poor performance when enthalpy losses due to pressure work in the expansion stroke became important. Therefore, they introduced a new variable volume tabulated homogeneous chemistry (VVTHC) approach to study diesel and HCCI engines [26]. The results for the pressure trace agreed with the experiment while prediction of the $\mathrm{CO}$ emission was still not satisfactory.

The probability density function (PDF) method has also been used to simulate HCCI conditions [27,28]. In this method, the PDF transport equation in the composition or velocity-composition space is solved using Eulerian fields or Lagrangian particles. Bisetti et al. [27] considered a spatially homogeneous transported PDF model to simulate HCCI conditions and compared the results against DNS. Using this model, they were able to capture the correct quantitative trend for the time history of the heat release rate, and first and second moments of temperature and hydroxyl radical mass fraction. Zhang et al. [28] used the PDF method to study the effect of turbulence/chemistry interaction in an HCCI engine. They used a hybrid particle/finite-volume method to solve the PDF transport equation. In spite of its good performance and generality, the computational cost for complex three-dimensional simulations with detailed chemistry is still very large compared to the aforementioned techniques. [28].

The conditional moment closure (CMC) model has also been used to simulate HCCI conditions [29]. Initially as a model for non-premixed flames, the CMC model was independently developed, by Klimenko [30] based on the joint PDF method and Bilger [31] based on the decomposition method. A set of transport equations for the conditional moments of some reactive scalars are solved. The equations are closed in the first order CMC model by assuming the conditional fluctuations to be small.

Over the past decade, the CMC model has been successfully applied to numerous non-premixed applications such as bluff body flames [32,33,34], jet flames $[35,36,37,38]$, hood fires [39,40], spray ignition [41], diesel engines [42, 
$43,44,45]$ and soot formation [46]. However, fewer studies have been performed in the context of premixed combustion [47,48,49].

For premixed combustion applications, the choice of a scalar as a conditioning variable which minimise the conditional fluctuations is a crucial task. In non-premixed combustion, mixture fraction (indicating local fraction of mass which emanated from the fuel stream), is the natural choice [50]. Mixturefraction has the advantage of being a conserved scalar whose PDF and scalar dissipation rate can be estimated with reasonable confidence. However defining a conditioning variable in premixed flames is more challenging. It is very case dependent and has been defined based on the sensible enthalpy, temperature and fuel or product mass fraction. One of the key challenges for premixed combustion $\mathrm{CMC}$ is that with these choices of conditioning variable, it becomes difficult to specify the scalar dissipation rate and the conditioning variable's PDF. This is because these variables are not passive scalars. They have a strong chemical source term which results in the appearance of thin premixed combustion fronts that are governed by reaction-diffusion balances. The structure of these fronts affects the scalar dissipation rate and the PDF, making them difficult to model, in contrast to the equivalent quantities for non-reacting scalars which can be readily determined from large-scale quantities.

As discussed earlier in this section, since HCCI is by definition a premixed mode of combustion, it is possible that thin premixed deflagration fronts can arise which are controlled by reaction-diffusion balances. For the reasons mentioned above, these structures are problematic for CMC-based closures. Whether or not these structures arise in HCCI has been studied by theory [51] and as mentioned earlier by DNS $[8,9,13]$. These studies show that the speed of ignition fronts is controlled by the gradient of ignition delay, and if this speed is comparable to the laminar flame speed, then premixed deflagrations can occur. However, as discussed in the literature, temperature fluctuations in HCCI engines have a magnitude about $25 \mathrm{~K}$ [5]. In these conditions gradients of ignition delay are believed to be insufficient to result in significant amounts of burning in premixed flames, at least away from walls, such that the predominant mode of combustion is a chemically controlled, spontaneous ignition [8, $9,13,14]$. As a result of the dominance of spontaneous ignition, the combustion process is mainly controlled by thermochemical state prior to ignition. In this situation, total enthalpy can be used as a conditioning variable. Although total enthalpy involves a source term, it spatially fluctuates only by compressible effects, which are small away from knocking conditions. (Knocking is anyway an undesirable operating condition.)

In our previous work [29], a CMC model based on a normalised enthalpy as the conditioning variable was developed to investigate effects of the thermal stratifications under HCCI conditions. The model was spatially zerodimensional and therefore is an affordable approach which could be used in a practical design process. To the extent that the model's assumptions are valid, it could also be used for more detailed investigations to understand thermal stratification effects in HCCI. The model was evaluated using two-dimensional DNSs of a lean $n$-heptane/air mixture with two different mean temperatures 
within the NTC region where two-stage ignition occurred and having different levels of thermal stratification [13]. The results showed an excellent performance of the CMC model in cases with a low level of thermal stratifications whereas the performance deteriorated as the level of stratification increased. It was shown that the presence of large conditional fluctuations as a result of the predominant deflagration combustion mode was the reason for the weak performance of the CMC model at the highly stratified condition.

The present paper differs from our previous study [29] in that we consider cases in which ignition occurs in a single stage, i.e. iso-octane/air mixtures [14], or there is only a weak two-stage ignition, i.e. $n$-heptane/air mixtures with the mean temperature of $1008 \mathrm{~K}$ [13]. The main motivation of considering these cases in the present paper is to comparatively study the performance of the CFD-CMC solver for different mixtures under various conditions. In particular, anticipating the influence of the gradient of ignition delay, we expect that the temperature-dependence of ignition delay, which is markedly different in cases which have two-stage ignition and cases that have one-stage ignition, will have a strong influence on the model performance. Additionally, in order to better understand the sources of conditional fluctuations, we also develop the second conditional moment equations in the context of premixed combustion, which is not reported in the literature to the best of our knowledge. Although this could ultimately be used in a second order closure, the second moment equations here are only used to identify the sources of conditional fluctuations. The contribution of the source terms is then investigated under different conditions.

\section{Conditional Moment Closure (CMC)}

To obtain the CMC equations, a normalised scalar variable is defined as:

$$
\theta(x, t)=\frac{h(x, t)-h_{\min }(t)}{h_{\max }(t)-h_{\min }(t)},
$$

where $h$ is the total enthalpy (chemical plus sensible) while $h_{\min }$ and $h_{\max }$ correspond to minimum and maximum enthalpies in the computational domain, respectively. The conditionally Favre-averaged mass fraction of species $\alpha, Y_{\alpha}$, as a function of the conditioning variable at time $t$ and location $x$ is then defined as

$$
Q_{\alpha}(\xi ; x, t)=\left\langle\left. Y_{\alpha}\right|_{\theta=\xi}\right\rangle=\frac{\overline{\left.\rho Y_{\alpha}(x, t)\right|_{\theta=\xi}}}{\overline{\left.\rho\right|_{\theta=\xi}}},
$$

where $\rho$ is the density and $\xi$ is the sample space for normalised enthalpy. The symbols $\overline{\left.()\right|_{\theta=\xi}}$ and $\left\langle\left.()\right|_{\theta=\xi}\right\rangle$ refer to the conditional mean and the Favreconditional mean, respectively. In the following, () $\left.\right|_{\theta=\xi}$ is shown by () $\left.\right|_{\xi}$.

Using the above definition, the species mass fraction equations, can be recast into a set of $\mathrm{CMC}$ transport equations by the decomposition method 
presented in Ref. [50],

$$
\begin{aligned}
\rho \dot{W}_{\alpha} & =\rho \frac{\partial Q_{\alpha}}{\partial t}+\rho v_{j} \frac{\partial Q_{\alpha}}{\partial x_{j}}+\rho S_{\theta} \frac{\partial Q_{\alpha}}{\partial \xi}-\rho N \frac{\partial^{2} Q_{\alpha}}{\partial \xi^{2}} \\
& -\frac{\partial}{\partial x_{j}}\left(\rho D \frac{\partial Q_{\alpha}}{\partial x_{j}}\right)-\rho D \frac{\partial \theta}{\partial x_{j}}\left(\frac{\partial}{\partial x_{j}} \frac{\partial Q_{\alpha}}{\partial x_{j}}\right) \\
& +\rho \frac{\partial Y_{\alpha}^{\prime \prime}}{\partial t}+\rho v_{j} \frac{\partial Y_{\alpha}^{\prime \prime}}{\partial x_{j}}-\frac{\partial}{\partial x_{j}}\left(\rho D \frac{\partial Y_{\alpha}^{\prime \prime}}{\partial x_{j}}\right)
\end{aligned}
$$

where $v_{j}$ is the velocity in the $x_{j}$ direction, $Y_{\alpha}^{\prime \prime}$ is the conditional mass fraction fluctuation of species $\alpha$ and $\dot{W}_{\alpha}$ is $\dot{\omega}_{\alpha} / \rho$, where $\dot{\omega}_{\alpha}$ is the reaction rate for species $\alpha$. The scalar dissipation rate, $N$, is defined as $D \frac{\partial \theta}{\partial x_{j}} \frac{\partial \theta}{\partial x_{j}}$, where $D$ is the molecular diffusivity, which is assumed to be equal to the thermal diffusivity. The term $S_{\theta}$ results from the source term of normalised enthalpy, defined as:

$$
S_{\theta}=\frac{1}{h_{\max }-h_{\min }}\left(\frac{1}{\rho} \frac{D p}{D t}-(1-\theta) \frac{\partial h_{\min }}{\partial t}-\theta \frac{\partial h_{\max }}{\partial t}\right) .
$$

where $p$ is the pressure. Proceeding, equation 3 is then conditionally averaged, the primary closure hypothesis is applied, and high Reynolds number is assumed [50]. In most CMC applications, this would leave spatial transport terms in the CMC. Here, however the focus is on the bulk gas region, which principally controls the rate of pressure rise and also emissions of $\mathrm{NO}_{\mathrm{x}}$, and it is assumed that in this region the flow has homogeneous conditional statistics. As per Ref. [29], we therefore ignore the spatial transport terms and the conditional moments evolve as:

$$
\frac{\partial Q_{\alpha}}{\partial t}=-v_{\xi} \frac{\partial Q_{\alpha}}{\partial \xi}+\left\langle\left. N\right|_{\xi}\right\rangle \frac{\partial^{2} Q_{\alpha}}{\partial \xi^{2}}+\left\langle\left.\dot{W}_{\alpha}\right|_{\xi}\right\rangle
$$

where $v_{\xi}$ is convective velocity in the $\xi$-space and is equal to $\left\langle\left. S_{\theta}\right|_{\xi}\right\rangle$ and $\left\langle\left.\dot{W}_{\alpha}\right|_{\xi}\right\rangle=\overline{\left.\dot{\omega}_{\alpha}\right|_{\xi}} / \overline{\left.\rho\right|_{\xi}}$. The conditional reaction rates can be obtained using the first order CMC approximation [29],

$$
\overline{\left.\dot{\omega}_{\alpha}\right|_{\xi}}=f\left(Q_{\alpha}, Q_{T}, \overline{\left.p\right|_{\xi}}\right)
$$

where $Q_{T}=\left\langle\left. T\right|_{\xi}\right\rangle$.

It is worth noting at this point that the final equations are the same as the flamelet equations presented in Cook et al. [19]. The connections between flamelet models and CMC models have been recognised for some time - as discussed comprehensively in Ref. [52]. The assumptions behind these models are connected but somewhat different. Cook et al. [19] used an order of magnitude argument to show that in deflagration dominated regions where enthalpy gradients are high, diffusion in directions tangential to enthalpy gradients could be neglected. In spontaneous ignition regions where enthalpy gradients are low, tangential terms can still be ignored even though their magnitudes are considerable in comparison to the non-tangential terms. This is due to the negligible molecular transport in spontaneous ignition regions. On the other hand 
the present analysis simply assumes the conditional fluctuations are small and does not assume any particular spatial structure. Nonetheless, the fact that the final equations are the same suggests that these assumptions are connected which provides mutual support for both modelling frameworks. The benefit of considering the CMC framework is that because it is a rigorous mathematical closure, the errors incurred by various assumptions can be quantified in terms of their overall effect on the model performance.

\section{Numerical methods and test cases studied}

The open source $\mathrm{C}++$ code known as OpenFOAM was employed to implement the CMC model presented in section 2. A flowchart demonstrating the solution algorithm is shown in Fig. 1. The CFD code implicitly solves the equations governing evolution of continuity, momenta and enthalpy for a compressible flow. It employs a pressure-implicit with splitting of operators (PISO) algorithm to treat the pressure-velocity coupling. After advancing the CFD code in time, the conditionally averaged normalised enthalpy source term, pressure and scalar dissipation rate are calculated and passed to the CMC code. In the next step, the conditional mass fraction equations, Eq. (5) are implicitly solved using an operator splitting method [41] which leads to two sets of equations: diffusion and convection, which are non-stiff, and the progress of chemical reactions, which are a set of stiff ordinary differential equations (ODEs). A semi-implicit Bullirsch-Stoer (SIBS) method is employed to solve the chemical reaction ODEs in $\xi$-space. After solving Eq. (5) the Favre-averaged species mass fractions on the CFD grid are obtained using an assumed Dirac-delta PDF for enthalpy. The use of the Dirac PDF is possible in the present case because the flow is deliberately resolved to a DNS level in terms of the conditioning variable, in order to focus only on the effects of the conditioning and not on the models for scalar dissipation and the conditioning variable PDF. (Of course, this approach is not affordable in a real engine, but being as the enthalpy source term does not fluctuate significantly in space, it is expected that standard models of its PDF and dissipation rate would be applicable ${ }^{1}$.) More details of the numerical methods can be found in Ref. [29].

Two sets of DNS data were used to examine the performance of CFDCMC solver. One DNS data-set modelled ignition of a lean $n$-heptane/air mixture with thermal stratifications in a constant volume [13] while the other modelled ignition of an iso-octane/air mixture in a similar configuration [14]. Both DNS databases were generated using S3D, a high order, fully explicit finite-difference solver which has been used for many studies $[53,54,55,56,57$, $58,59]$. A 58-species and a 99-species reduced chemical mechanism $[13,14]$ were used for the $n$-heptane and iso-octane cases, respectively. All the DNS cases were initialised with a uniform mixture composition field having equivalence

1 This is expected to be true in the bulk gas region but the near-wall region may require a different treatment. 
ratio of 0.3 and initial uniform pressures of $40 \mathrm{~atm}$ and $20 \mathrm{~atm}$ for the $n$ heptane and iso-octane cases, respectively. The main parameters of the DNS cases are shown in Table $1[13,14]$. The initial mean temperature is denoted $T_{0}$; the temperature fluctuation root mean square (RMS) $T^{\prime}$; the turbulence velocity fluctuation $u^{\prime}$ and most energetic turbulent length scale $l_{e}$. Three $n$ heptane DNS cases were considered having a mean temperature of $1008 \mathrm{~K}$, slightly outside the NTC regime, while five iso-octane cases were considered having a mean temperature of $1035 \mathrm{~K}$. In both cases, the temperatures are sufficiently high so that only a single stage ignition is observed, in contrast to our previous study that considered lower mean temperatures having a twostage ignition [29]. Temperature inhomogeneities were varied from 15 to $60 \mathrm{~K}$ for both fuels. As can be seen in Table 1, two last iso-octane cases, octane- 8 and octane-9, have the same level of thermal stratifications as case octane-1 whereas the turbulence intensity and integral length scale are different. The homogeneous ignition delay times, $\tau_{i g, 0}$, corresponding to the mean temperatures of $n$-heptane and iso-octane cases were very similar between the two cases at $2.49 \mathrm{~ms}$ [13] and 2.5 [14] ms, respectively.

The initial temperature fields for cases heptane-12 and octane-3 with identical temperature fluctuations of $60 \mathrm{~K}$ are shown in Fig. 2. As can be seen, the initial temperature distributions are similar in both cases; the slight difference is because of the different mean temperatures in cases heptane- 12 and octane-3.

A uniform two-dimensional CFD grid of $160 \times 160$ was employed for all cases, compared with the DNS grid of $640 \times 640$. Note that a coarser mesh can be used for the CMC-based model since only the length scales of enthalpy and velocity fluctuations need to be resolved, while the DNS needed to resolve species that fluctuate on smaller length scales. The CMC conditioning variable domain included 101 equally spaced grid points. The time-step used in the CFD-CMC solver was $2.5 \mu$ s while the DNS time-step was $2.5 \mathrm{~ns}$. The initial and boundary conditions for the CFD-CMC were the same as those in the DNS cases.

\section{Results}

\subsection{Comparison of CFD-CMC results and DNS}

Figure 3 presents the mean heat release rate and pressure versus time obtained from the CFD-CMC solver in comparison with the DNS data. Time is normalised by the homogeneous ignition delay times of 2.49 and $2.5 \mathrm{~ms}$ for $n$-heptane and iso-octane cases, respectively. As can be seen, the predicted mean values from the CFD-CMC solver are in excellent agreement with the DNS data for all $n$-heptane cases with temperature fluctuations of $15-60 \mathrm{~K}$, in agreement with the observations in Ref. [29] for lower mean temperatures where strong two-stage ignition was observed, demonstrating that good agreement is not limited to conditions having two-stage ignition. On the other hand, 
the CMC model predicts the ignition delay time and the peak heat release rate with good accuracy for the iso-octane cases with $T^{\prime}=15$ and $30 \mathrm{~K}$ whereas for the case with a high level of temperature inhomogeneities (case octane-3 with $T^{\prime}=60 \mathrm{~K}$ ), the agreement is not as good as the other iso-octane cases (but still reasonable).

It is interesting to note that the CMC model predicts the ignition delay time and the peak heat release rate with a good accuracy for case heptane-12 with $T^{\prime}=60 \mathrm{~K}$ whereas for case octane-3 with the same level of temperature inhomogeneities, ignition occurs earlier than in the DNS. This demonstrates that the level of temperature fluctuations alone cannot be used as a performance indicator for the CFD-CMC solver.

To investigate the difference between cases heptane- 12 and octane- 3 , it is important to understand the differences in ignition characteristics between $n$ heptane and iso-octane/air mixtures. To do so, a series of cases modelling ignition in homogeneous $n$-heptane/air and iso-octane/air mixtures at a constant volume were simulated for a range of initial temperatures from $800 \mathrm{~K}$ to 1250 $\mathrm{K}$ with equivalence ratio and pressure corresponding to the initial conditions of $2 \mathrm{D}$ cases. The simulations were performed using the same DNS code as the original DNS. Figure 4 presents the ignition delay times versus the reciprocal of the temperature for both $n$-heptane/air and iso-octane/air mixtures. It is readily observed that the iso-octane exhibits a positive temperature coefficient of ignition delay whereas the $n$-heptane exhibits NTC behaviour towards the lower temperature range. Over the range of temperatures considered, a larger variation of ignition delay time is consequently observed for iso-octane compared with $n$-heptane. As a result, the $2 \mathrm{D}$ iso-octane cases feature a higher level of ignition delay time fluctuations.

Using a mapping between the initial temperature (shown in Fig. 2) at a point and the ignition delay obtained for homogeneous conditions at a given temperature and at constant volume (shown in Fig. 4) the distribution of ignition delay times in the 2D cases can be obtained. Figure 5 shows this distribution for cases heptane-12 and octane-3. As can be seen, the level of ignition delay time fluctuations in case octane- 3 with $T^{\prime}=60 \mathrm{~K}$ is much higher than that in case heptane-12 which has the same level of thermal stratifications. This is consistent with the results presented in Fig. 4.

Table 2 presents the ignition delay time fluctuation RMS, $\tau_{i g}^{\prime}$,

$$
\tau_{i g}^{\prime}=\sqrt{\int_{A} \frac{\left(\tau_{i g}^{i}-\bar{\tau}_{i g}\right)^{2} d A}{A}},
$$

where $\bar{\tau}_{i g}=\int_{A} \tau_{i g}^{i} d A / A$ and $A$ is the domain area. The variable $\tau_{i g}^{i}$ is the initial ignition delay time distribution at a given point (e.g. shown in Fig. 5 for case octane-3) for all $n$-heptane and iso-octane cases. All iso-octane cases have larger $\tau_{i g}^{\prime}$ compared to those in $n$-heptane cases with the same level of thermal stratification. This is again consistent with the results presented in Fig. 4, as iso-octane shows a stronger ignition delay time dependency on temperature compared with $n$-heptane. This finding as well as our observation 
in Fig. 3 suggests that there is a correlation between the performance of the CFD-CMC solver and RMS of the ignition delay time at the initial conditions. More discussion of this point will provided later in this paper.

The ignition delay time, $\tau_{i g}$, burning duration, $\tau_{b}$, and maximum mean heat release rate, $\mathrm{HRR}_{\max }$, obtained from the CFD-CMC solver are also compared with the DNS results in Table 2 for all cases. The burning duration is defined as the full width at half maximum of the mean heat release rate. As can be seen, there is a good agreement between the CFD-CMC results and DNS for the burning duration. However, for the ignition delay time and peak heat release rate, the agreement is worst for the highest $\tau_{i g}^{\prime}$.

Figure 6 presents error percentages of the peak heat release rate and ignition delay time versus $\tau_{i g}^{\prime}$ normalised by $\tau_{i g}$ for all cases in comparison to the DNS results. The errors are also presented in Table 2. Figure 6a shows that on average, there is an increasing trend for the error percentage as a function of normalised $\tau_{i g}^{\prime}$. This therefore suggests that the normalised $\tau_{i g}^{\prime}$ can be used as an indicator for the performance of the CMC model. Figure $6 \mathrm{~b}$, however, does not show a similar trend. Nevertheless, one should note that for all cases with the exception of case octane-3, the error percentage is less than $2 \%$. This therefore suggests that the CMC model can predict the ignition delay time with a good accuracy for a large range of ignition delay time fluctuations.

It is also observed that case octane- 8 exhibits a lower error value for the peak heat release rate compared with the case octane-1. The only difference between these two cases is that case octane- 8 has a larger $u^{\prime}$. As discussed in Ref. [14], the turbulence has a key role to homogenise the mixture. Therefore, higher level of homogenisation in octane- 8 leads to the initial $\tau_{i g}^{\prime}$ reducing more quickly and less error percentage in the prediction of the peak heat release rate. Comparison of cases octane- 9 and 1, featuring the same level of velocity fluctuations, shows worse agreement for octane- 9 with a lower initial $l_{e}$ and a shorter turbulence time scale, defined as $\tau_{t}=l_{e} / u^{\prime}$. This may at first seem counterintuitive. However, as discussed by Yoo et al. [14], since $l_{e}$ in case octane- 9 is smaller than the length scale of temperature fluctuations $\left(l_{e} / l_{T_{e}}=0.2\right)$ a shorter turbulence time scale in case octane- 9 compared with case octane- 1 does not lead to a higher level of mixture homogenisation. Therefore, a better agreement for case octane- 9 is not expected although it has the same turbulence time scale as case octane- 8 .

Figure 7 presents isocontours of temperature obtained using the CFD-CMC solver for case octane- 3 with $T^{\prime}=60 \mathrm{~K}$ in comparison with the DNS data at $t / \tau_{i g}=0.3,0.45,0.63$ and 0.8 . As can be seen, there is an overall reasonable agreement between the CMC model and DNS. It can also be observed that ignition is advanced in some regions when the CMC model is used. For instance, regions A and B marked in Fig. 7 show a higher temperature predicted by the CMC model at $t / \tau_{i g}=0.63$ compared with that in DNS. These regions are surrounded by the isocontour of $\xi=0.66$ (solid black line) where the conditional fluctuations are high and therefore, the performance of the first CMC model is poor. Conditional mass fractions of fuel for case octane-3 obtained from the CFD-CMC solver at different instants are also compared 
with the DNS results in Fig. 8. It can be observed that at $t / \tau_{i g}=0.3$ and 0.46 , the CFD-CMC and DNS results are in agreement whereas at the later time ignition is advanced in the CFD-CMC results for the regions of higher enthalpy.

In the next section, additional analysis of the DNS data will shed light on the reasons for disagreement between the CMC model and DNS.

\subsection{Analysis of the DNS data}

\subsubsection{Conditional fluctuations}

Figure 9 shows scatter plots of fuel mass fraction versus the conditioning variable, $\xi$, at several instants for cases heptane-12 and octane- 3 featuring the highest level of temperature fluctuations. The scatter plots are coloured by the logarithm of scalar dissipation rate, $\log _{10}(N)$.

As can be seen, in case heptane-12 ignition occurs in both regions with low and high enthalpies, then propagates towards the unburned region. The appearance of ignition kernels in both high and low enthalpy regions is due to the NTC behaviour observed in case heptane-12 even though the mean temperature of $1008 \mathrm{~K}$ is outside the NTC regime. In case octane-3, ignition starts from the regions with high enthalpies and then propagates into the regions of low enthalpies. As can be seen, in both cases, due to the importance of deflagrative modes of combustion $[13,14]$, conditional fluctuations are significant. Nevertheless, there is a good agreement between CFD-CMC and DNS for the mean quantities for case heptane-12. This observation therefore suggests that large conditional fluctuations do not necessarily imply poor performance of the CMC model.

As found in Ref. [29], dissipation rate fluctuations can also be the cause of disagreement between the CMC model and the DNS results in cases with a high level of thermal stratification. As shown in Fig. 9, in both cases heptane-12 and octane-3, dissipation rate fluctuations are correlated with the conditional fluctuations. However, case octane-3 exhibits a stronger correlation. As a result, the disagreement between the CMC model and the DNS data observed in case octane- 3 may be due to this strong correlation.

Correlation factors defined by Pope [60] between dissipation rate and fuel mass fraction for both cases heptane- 12 and octane- 3 at $t / \tau_{i g}=0.3,0.45$, 0.63 and 0.8 , are also shown in Fig. 10. It can be seen that both cases present mostly a negative correlation. This indicates that ignition is enhanced in regions with higher scalar dissipation, and (relatively) retarded in regions having a lower dissipation rate. Figure 10 also demonstrates that there is a stronger correlation in case octane- 3 compared with case heptane- 12 , hence the firstorder CMC model representing only mean scalar dissipation rate is not able to predict ignition process accurately in the former case.

Figure 11 presents the local conditional dissipation rate fluctuations, i.e. 
$\log _{10} N-\log _{10}\left\langle\left. N\right|_{\xi}\right\rangle$ and the local conditional fluctuations of fuel mass fraction, i.e. $Y_{\alpha}-\left\langle\left. Y_{\alpha}\right|_{\xi}\right\rangle$, respectively, along the isocontour of $\xi=0.66$ where the conditional fluctuations are significant in case octane-3 at $t / \tau_{i g}=0.63$. It can be seen that both conditional dissipation and mass fraction fluctuations are significant along the isocontour of $\xi=0.66$. It can be observed that the positive conditional fluctuations of the fuel mass fraction are often correlated with the negative conditional fluctuations of dissipation rate and vice versa. Due to the importance of both conditional dissipation rate and mass fraction fluctuations, the accuracy of the CMC model is not satisfactory in case octane-3. This will be discussed further in following sections.

\subsubsection{Evaluation of the first order closure}

To investigate the performance of the first CMC closure in case octane- 3 with $T^{\prime}=60 \mathrm{~K}$, the conditional closures appearing in Eq. (5) are evaluated using the DNS data. To do so, all conditional terms as well as the PDF of the normalised enthalpy, $P(\xi)$, are obtained directly using the DNS data; details of this test can be found in Ref. [29]. Figure 12a presents the trace of the mean $\mathrm{CO}_{2}$ mass fraction compared to the DNS for case octane-3. The excellent agreement confirms that the CMC equation, i.e. Eq. (5), is closed. This leads us to conclude that the primary closure hypothesis is not the reason for the discrepancy, and moreover provides verification of the numerical implementation of the CMC model.

To further investigate the first-order closure hypothesis, an a priori test similar to what presented in Ref. [29] is defined. In this test, conditional mean reaction rates directly obtained using the DNS data are compared with the conditional reaction rates determined by feeding the DNS conditional averages $Q_{\alpha}$ and $Q_{T}$ to the first order closure $\dot{\omega}_{\alpha}\left(Q_{\alpha}, Q_{T}\right)$. The results are presented in Fig. 12b, which illustrates the mean $\mathrm{CO}_{2}$ reaction rate versus time normalised by the ignition delay time for case octane- 3 with $T^{\prime}=60 \mathrm{~K}$. As can be seen, the first order closure over predicts the reaction rate of $\mathrm{CO}_{2}$ across all enthalpies, particularly near the peak reaction rate.

\subsubsection{Evaluation of the conditional variance}

To further investigate the cause of the conditional fluctuations, the conditional expectations of the second moments of reactive scalars [50] are considered in the following. The conditional covariance $G_{\alpha \beta}$ of species $\alpha$ and $\beta$ is defined as $G_{\alpha \beta}=\left\langle\left. K_{\alpha \beta}\right|_{\xi}\right\rangle$ where $K_{\alpha \beta}=Y_{\alpha}^{\prime \prime} Y_{\beta}^{\prime \prime}$. The variable $Y_{\alpha}^{\prime \prime}$, the conditional mass fraction fluctuation of species $\alpha$, is defined as $Y_{\alpha}-\left\langle\left. Y_{\alpha}\right|_{\xi}\right\rangle$. The conditional covariance/variance equations for non-premixed combustion have been investigated in the literature $[50,61,62,63,64,65]$; however, to the best of our knowledge, the conditional covariance/variance equations for premixed flames has not been reported. Here, an equation for the conditional variance, $G_{\alpha \alpha}$, using the decomposition method [50] with the assumption of statistically homogeneous charge is derived from Eq. (3). For the details of the derivation, 
the reader is referred to appendix A. By neglecting the spatial derivatives, the equation for the conditional variance may be written as follows,

$$
\begin{aligned}
\frac{\partial G_{\alpha \alpha}}{\partial t} & =\left\langle\left. N\right|_{\xi}\right\rangle \frac{\partial^{2} G_{\alpha \alpha}}{\partial \xi^{2}}-\left\langle\left. S_{\theta}\right|_{\xi}\right\rangle \frac{\partial G_{\alpha \alpha}}{\partial \xi} \\
& +2\left\langle\left.\dot{W}_{\alpha}^{\prime \prime} Y_{\alpha}^{\prime \prime}\right|_{\xi}\right\rangle+2\left\langle\left. N^{\prime \prime} Y_{\alpha}^{\prime \prime}\right|_{\xi}\right\rangle \frac{\partial^{2} Q_{\alpha}}{\partial \xi^{2}}-2\left\langle\left. S_{\theta}^{\prime \prime} Y_{\alpha}^{\prime \prime}\right|_{\xi}\right\rangle \frac{\partial Q_{\alpha}}{\partial \xi},
\end{aligned}
$$

where ()$^{\prime \prime}=()-\left\langle\left.()\right|_{\xi}\right\rangle$. The source terms in Eq. (8) consist of $\left\langle\left.\dot{W}_{\alpha}^{\prime \prime} Y_{\alpha}^{\prime \prime}\right|_{\xi}\right\rangle$ representing the correlation between reaction rate and mass fraction fluctuations, $\left\langle\left. N^{\prime \prime} Y_{\alpha}^{\prime \prime}\right|_{\xi}\right\rangle \frac{\partial^{2} Q_{\alpha}}{\partial \xi^{2}}$ representing the correlation between dissipation rate and mass fraction fluctuations and $\left\langle\left. S_{\theta}^{\prime \prime} Y_{\alpha}^{\prime \prime}\right|_{\xi}\right\rangle \frac{\partial Q_{\alpha}}{\partial \xi}$ representing the correlation between fluctuations of the source term in the conditioning variable transport equation and mass fraction fluctuations. Compared with the conditional covariance equation for a conserved scalar, the new terms are the conditional mean source, which is an advective term in conditioning space, and the source-term mass-fraction correlation.

The conditional variance for fuel obtained using the integration of the right and left hand sides of Eq. (8) for case octane-3 is shown in Fig. 13 at different instants. The derivatives with respect to $\xi$ appeared in Eq. (8) are obtained by fitting a ninth-order polynomial to the appropriate conditional mean and covariance. Swaminathan and Bilger [47,62] showed that this method was able to capture the derivatives accurately. Figure 13 shows that Eq. (8) is closed when all terms are provided by the DNS.

As shown in Fig. 13, not surprisingly, at early stage of ignition, the conditional variance is small while at later stage it becomes substantial. It can be seen that from $t / \tau_{i g}=0.3$ to $t / \tau_{i g}=0.8$, the conditional variance becomes considerable for a wider range of normalised enthalpies, which is consistent with the observation in Fig. 9. It is interesting to note that as the peak of the conditional variance moves from the region of high to low enthalpies, conditional fluctuations appear in a wider region in the enthalpy domain. This can be due to the increasing contribution of the deflagration mode as the flame propagates into the unburned region. Once deflagrations appear, they can propagate in directions which are not necessarily aligned with the conditioning variable, thus generating more conditional fluctuations. Eventually however, conditional variance is destroyed in the burned regions as equilibrium is approached.

Figure 14 presents $\left\langle\left.\dot{W}_{\alpha}^{\prime \prime} Y_{\alpha}^{\prime \prime}\right|_{\xi}\right\rangle,\left\langle\left. N^{\prime \prime} Y_{\alpha}^{\prime \prime}\right|_{\xi}\right\rangle$ and $\left\langle\left. S_{\theta}^{\prime \prime} Y_{\alpha}^{\prime \prime}\right|_{\xi}\right\rangle$ at $t / \tau_{i g}=0.8$ for fuel in cases octane- 1 with $T^{\prime}=15 \mathrm{~K}$ and octane- 3 with $T^{\prime}=60 \mathrm{~K}$. There are several points to be made here. First, all source terms are negligible in case octane-1 with low level of thermal stratification when compared with case octane-3 with a large level of temperature inhomogeneities. It can be therefore concluded the first order CMC model is accurate for cases with low level of thermal stratification, as already noted in the earlier a posteriori tests. Second, the term due to $S_{\theta}$-fluctuations is relatively small in both cases octane- 1 and 
octane-3. (It is important to note, however, that this is due to the choice of total enthalpy as a conditioning variable, since as mentioned earlier, the fluctuations of its source term are acoustically scaled and hence small in the present non-knocking conditions. For conventional premixed combustion closures, in which a progress-variable may be used as the conditioning variable, this term could well be quite significant.) Coming back to the main argument, the third point is that source terms due to reaction rate fluctuations and $N$-fluctuations have the same order of magnitude in case octane-3. This is consistent with the observation in Fig. 9, demonstrating a strong correlation between dissipation rate fluctuations and conditional fluctuations in case octane-3. Fourth, the source term due to $N$-fluctuations is negative across all normalised enthalpies which is in agreement with dominant negative correlation found in Fig. 10d. Finally, the reaction rate fluctuations reveal a positive correlation with the conditional fluctuations in a significant part of the burning regions.

To shed more light on the cause of the conditional fluctuations observed in case octane-3 with large thermal stratifications, all terms on the right hand side of Eq. (8) for fuel mass fraction variance obtained from the DNS data are presented at different instants in Fig. 15. As discussed previously, since $\left\langle\left. S_{\theta}^{\prime \prime} Y_{\alpha}^{\prime \prime}\right|_{\xi}\right\rangle$ is small at all instants, the source term due to these fluctuations is small as well. The contribution of the convective term, $\left\langle\left. S_{\theta}\right|_{\xi}\right\rangle \frac{\partial G_{\alpha \alpha}}{\partial \xi}$, is also insignificant. This is because $S_{\theta}$ represents the effects of the temporal evolution of pressure in the normalised enthalpy domain (and not an un-normalised domain), where the rate of pressure rise term roughly cancels the change of the maximum to minimum enthalpy difference - c.f. Eq. (4). The importance of the diffusion term, $\left\langle\left. N\right|_{\xi}\right\rangle \frac{\partial^{2} G_{\alpha \alpha}}{\partial \xi^{2}}$, is also found to be minor in comparison with the source terms, $\left\langle\left.\dot{W}_{\alpha}^{\prime \prime} Y_{\alpha}^{\prime \prime}\right|_{\xi}\right\rangle$ and $\left\langle\left. N^{\prime \prime} Y_{\alpha}^{\prime \prime}\right|_{\xi}\right\rangle \frac{\partial^{2} Q_{\alpha}}{\partial \xi^{2}}$.

As can be seen, the term due to $N$-fluctuations acts as a source (positive values) in regions with low enthalpies while it acts as a sink in regions with high enthalpies; for instance at $t / \tau=0.8$ about $\xi=0.45$. This is consistent with the data presented in the previous sections. However, as can be seen in Fig. 10d and Fig. 14b, the correlation between conditional dissipation and mass fraction fluctuations embedded in this term is negative across all enthalpies. Therefore, the second derivative, $\frac{\partial^{2} Q_{\alpha}}{\partial \xi^{2}}$ should be negative and positive for $\xi<0.45$ and $\xi>0.45$, respectively. A similar trend for the second derivative, $\frac{\partial^{2} Q_{\alpha}}{\partial \xi^{2}}$, was also found in Ref. [19] for a thermally stratified $\mathrm{H}_{2}$ /air mixture. This term therefore effectively transports conditional fluctuations from burned to igniting regions. On the other hand, $\left\langle\left.\dot{W}_{\alpha}^{\prime \prime} Y_{\alpha}^{\prime \prime}\right|_{\xi}\right\rangle$ acts more prevalently as a production term, but also tends to destroy fluctuations somewhat in the burned gases. It can be seen that the terms due to $W$-fluctuations and $N$-fluctuations are respectively the most dominant and second-most dominant terms in Eq. (8), in particular at later stages of ignition (Note that the term due to $N$-fluctuations is about two times smaller than the source term due to $W$-fluctuations at $t / \tau_{i g}=0.8$ ). Another point to note is that the terms never balance across the whole of the enthalpy space, suggesting that transport equations would need to be solved for 
the conditional second moments rather than attempting to devise an algebraic model from a balance of modelled source terms.

Figure 16a shows the source terms, $\left\langle\left.\dot{W}_{\alpha}^{\prime \prime} Y_{\alpha}^{\prime \prime}\right|_{\xi}\right\rangle$ and $\left\langle\left. N^{\prime \prime} Y_{\alpha}^{\prime \prime}\right|_{\xi}\right\rangle \frac{\partial^{2} Q_{\alpha}}{\partial \xi^{2}}$ for fuel, which are found to be significant in Eq. (8) in cases heptane-12 and octane-3 at $t / \tau_{i g}=0.8$. As can be seen, even though case octane- 3 reveals a stronger correlation between dissipation fluctuations and conditional fluctuations (Fig. 10.d), term $\left\langle\left. N^{\prime \prime} Y_{\alpha}^{\prime \prime}\right|_{\xi}\right\rangle \frac{\partial^{2} Q_{\alpha}}{\partial \xi^{2}}$ in case heptane-12 is relatively larger. On the other hand, term $\left\langle\left.\dot{W}_{\alpha}^{\prime \prime} Y_{\alpha}^{\prime \prime}\right|_{\xi}\right\rangle$ is larger in case octane-3 compared with case heptane12. It also shows that these terms amplify each other, i.e. have the same sign, across all conditioning variables, $\xi$, in case octane- 3 whereas in case heptane-12 there is a region between around $\xi=0.45$ and $\xi=0.55$ where the dissipation term is positive while the reaction term is negative. Figure 16b shows the right hand side of the conditional variance equation, Eq. (8) for fuel in cases heptane- 12 and octane- 3 at $t / \tau_{i g}=0.8$. As can be seen, the right hand side of Eq. (8) in case octane-3 is larger than that of case heptane-12. In particular the integral of this term over the conditioning variable space is very much higher for the iso-octane case. This therefore explains the overall better performance of the CMC model in case heptane-12 relative to case octane-3.

\section{Conclusions}

The CMC model presented in Ref. [29] was applied to two sets of DNS data featuring thermal stratifications in lean $n$-heptane/air and iso-octane/air mixtures with mean temperatures outside of the negative temperature coefficient regime. The results for all $n$-heptane cases with the mean temperature of 1008 $\mathrm{K}$ and temperature fluctuations of $15-60 \mathrm{~K}$ show excellent agreement with the DNS data. The results for the iso-octane cases with $T^{\prime}=15$ and $30 \mathrm{~K}$ show an excellent performance of the CFD-CMC solver while the results for case octane-3 with $T^{\prime}=60 \mathrm{~K}$ show some departure. A series of homogeneous ignition simulations corresponding to the initial conditions of the two-dimensional cases revealed a stronger dependency of ignition delay time to the temperature in the iso-octane/air mixture compared with the $n$-heptane/air mixture. As a result, the level of ignition delay time fluctuations, $\tau_{i g}^{\prime}$, is much higher in iso-octane cases in comparison with those in $n$-heptane cases with the same level of thermal stratification. Case octane- $3\left(T^{\prime}=60 \mathrm{~K}\right)$ with the highest level of ignition delay time fluctuations, presented maximum percentages of error for the peak heat release rate and the ignition delay time of $9.7 \%$ and $12.5 \%$, respectively.

To investigate the cause of conditional fluctuations, the transport equation for the conditional variance was derived and studied using the DNS data. An assessment of conditional variance equation for case octane- 1 with a low level of thermal stratifications $\left(T^{\prime}=15 \mathrm{~K}\right)$ showed that source terms to generate conditional fluctuations were negligible. This was consistent with the observed small conditional fluctuations in case octane- 1 and the good performance of the a posteriori test of the first order closure model. On the other hand, 
the source term due to the correlation between mass fraction and dissipation rate fluctuations and the source term due to the correlation between mass fraction and reaction rate fluctuations were found to be the cause of significant conditional fluctuations in case octane- 3 with a large thermal stratification level. These terms became significant in the later stages of ignition, leading to an increased conditional fluctuations.

Further examination of the DNS data revealed that there was a stronger correlation between dissipation rate fluctuations and conditional mass fraction fluctuations in case octane-3 compared with case heptane-12. It was interesting to note that although both source terms due to the dissipation rate and reaction rate fluctuations were found larger in case heptane-12, their overall effects were much lower compared with case octane-3. This observation may be traced to the significantly higher temperature dependence of reaction rates to temperature in the iso-octane cases (as evidenced by ignition delay times).

Acknowledgements The research benefited from computational resources provided through the National Computational Merit Allocation Scheme, supported by the Australian Government. The computational facilities supporting this project included the Australian NCI National Facility, the partner share of the NCI facility provided by Intersect Australia Pty Ltd., the Peak Computing Facility of the Victorian Life Sciences Computation Initiative (VLSCI), iVEC (Western Australia), and the UNSW Faculty of Engineering. This work was supported by the Australian Research Council (ARC).

\section{Appendix}

A derivation of the conditional variance equation for premixed combustion using the decomposition method [50] is presented here. It is assumed that all species have the same molecular diffusivity, which is equal to the thermal diffusivity.

Multiplying Eq. (3) for species $\alpha$ and $\beta$ by $Y_{\beta}^{\prime \prime}$ and $Y_{\alpha}^{\prime \prime}$, respectively and then adding them together leads to the following equation:

$$
\begin{aligned}
\rho Y_{\beta}^{\prime \prime} \frac{\partial Y_{\alpha}^{\prime \prime}}{\partial t}+\rho Y_{\alpha}^{\prime \prime} \frac{\partial Y_{\beta}^{\prime \prime}}{\partial t} & =-\rho v_{j} Y_{\beta}^{\prime \prime} \frac{\partial Y_{\beta}^{\prime \prime}}{\partial x_{j}}-\rho v_{j} Y_{\alpha}^{\prime \prime} \frac{\partial Y_{\alpha}^{\prime \prime}}{\partial x_{j}} \\
& +Y_{\beta}^{\prime \prime} \frac{\partial}{\partial x_{j}}\left(\rho D \frac{\partial Y_{\alpha}^{\prime \prime}}{\partial x_{j}}\right)+Y_{\alpha}^{\prime \prime} \frac{\partial}{\partial x_{j}}\left(\rho D \frac{\partial Y_{\beta}^{\prime \prime}}{\partial x_{j}}\right) \\
& -\rho Y_{\beta}^{\prime \prime} \frac{\partial Q_{\alpha}}{\partial t}-\rho Y_{\alpha}^{\prime \prime} \frac{\partial Q_{\beta}}{\partial t} \\
& -\rho v_{j} Y_{\beta}^{\prime \prime} \frac{\partial Q_{\alpha}}{\partial x_{j}}-\rho v_{j} Y_{\alpha}^{\prime \prime} \frac{\partial Q_{\beta}}{\partial x_{j}} \\
& -\rho S_{\theta} Y_{\beta}^{\prime \prime} \frac{\partial Q_{\alpha}}{\partial \xi}-\rho S_{\theta} Y_{\alpha}^{\prime \prime} \frac{\partial Q_{\beta}}{\partial \xi} \\
& +\rho N Y_{\beta}^{\prime \prime} \frac{\partial^{2} Q_{\alpha}}{\partial \xi^{2}}+\rho N Y_{\alpha}^{\prime \prime} \frac{\partial^{2} Q_{\beta}}{\partial \xi^{2}}
\end{aligned}
$$




$$
\begin{aligned}
& +Y_{\beta}^{\prime \prime} \frac{\partial}{\partial x_{j}}\left(\rho D \frac{\partial Q_{\alpha}}{\partial x_{j}}\right)+Y_{\alpha}^{\prime \prime} \frac{\partial}{\partial x_{j}}\left(\rho D \frac{\partial Q_{\beta}}{\partial x_{j}}\right) \\
& +\rho D Y_{\beta}^{\prime \prime} \frac{\partial \theta}{\partial x_{j}}\left(\frac{\partial}{\partial x_{j}} \frac{\partial Q_{\alpha}}{\partial \xi}\right)+\rho D Y_{\alpha}^{\prime \prime} \frac{\partial \theta}{\partial x_{j}}\left(\frac{\partial}{\partial x_{j}} \frac{\partial Q_{\beta}}{\partial \xi}\right) \\
& +\rho \dot{W}_{\alpha} Y_{\beta}^{\prime \prime}+\rho \dot{W}_{\beta} Y_{\alpha}^{\prime \prime} .
\end{aligned}
$$

If covariance $K_{\alpha \beta}$ of species $\alpha$ and $\beta$ is defined as $K_{\alpha \beta}=Y_{\alpha}^{\prime \prime} Y_{\beta}^{\prime \prime}$, Eq. (A.1) can be rearranged as:

$$
\begin{aligned}
\rho \frac{\partial K_{\alpha \beta}}{\partial t} & =-\rho v_{j} \frac{\partial K_{\alpha \beta}}{\partial x_{j}} \\
& +\frac{\partial}{\partial x_{j}}\left(\rho D \frac{\partial K_{\alpha \beta}}{\partial x_{j}}\right)-2 \rho D\left(\frac{\partial Y_{\alpha}^{\prime \prime}}{\partial x_{j}} \frac{\partial Y_{\beta}^{\prime \prime}}{\partial x_{j}}\right) \\
& -\rho Y_{\beta}^{\prime \prime} \frac{\partial Q_{\alpha}}{\partial t}-\rho Y_{\alpha}^{\prime \prime} \frac{\partial Q_{\beta}}{\partial t} \\
& -\rho v_{j} Y_{\beta}^{\prime \prime} \frac{\partial Q_{\alpha}}{\partial x_{j}}-\rho v_{j} Y_{\alpha}^{\prime \prime} \frac{\partial Q_{\beta}}{\partial x_{j}} \\
& -\rho S_{\theta} Y_{\beta}^{\prime \prime} \frac{\partial Q_{\alpha}}{\partial \xi}-\rho S_{\theta} Y_{\alpha}^{\prime \prime} \frac{\partial Q_{\beta}}{\partial \xi} \\
& +\rho N Y_{\beta}^{\prime \prime} \frac{\partial^{2} Q_{\alpha}}{\partial \xi^{2}}+\rho N Y_{\alpha}^{\prime \prime} \frac{\partial^{2} Q_{\beta}}{\partial \xi^{2}} \\
& +Y_{\beta}^{\prime \prime} \frac{\partial}{\partial x_{j}}\left(\rho D \frac{\partial Q_{\alpha}}{\partial x_{j}}\right)+Y_{\alpha}^{\prime \prime} \frac{\partial}{\partial x_{j}}\left(\rho D \frac{\partial Q_{\beta}}{\partial x_{j}}\right) \\
& +\rho D Y_{\beta}^{\prime \prime} \frac{\partial \theta}{\partial x_{j}}\left(\frac{\partial}{\partial x_{j}} \frac{\partial Q_{\alpha}}{\partial x_{j}}\right)+\rho D Y_{\alpha}^{\prime \prime} \frac{\partial \theta}{\partial x_{j}}\left(\frac{\partial}{\partial x_{j}} \frac{\partial Q_{\beta}}{\partial x_{j}}\right) \\
& +\rho \dot{W}_{\alpha} Y_{\beta}^{\prime \prime}+\rho \dot{W}_{\beta} Y_{\alpha}^{\prime \prime} .
\end{aligned}
$$

The covariance of species $\alpha$ and $\beta$ can be decomposed to the conditional covariance, $G_{\alpha \beta}$, and its fluctuations, $K_{\alpha \beta}^{\prime \prime}$ :

$$
K_{\alpha \beta}=G_{\alpha \beta}+K_{\alpha \beta}^{\prime \prime},
$$

where using definition $\left\langle K_{\alpha \beta}^{\prime \prime} \mid \xi\right\rangle=0$. Using Eq. (A.3), the temporal derivative at the left side of Eq. (A.2) can be written as:

$$
\frac{\partial K_{\alpha \beta}}{\partial t}=\frac{\partial G_{\alpha \beta}}{\partial t}+\frac{\partial G_{\alpha \beta}}{\partial \xi} \frac{\partial \theta}{\partial t}+\frac{\partial K_{\alpha \beta}^{\prime \prime}}{\partial t},
$$

and also the first and second terms on the right hand side of Eq. (A.2) can be described as follows:

$$
\frac{\partial K_{\alpha \beta}}{\partial x_{j}}=\frac{\partial G_{\alpha \beta}}{\partial x_{j}}+\frac{\partial G_{\alpha \beta}}{\partial \xi} \frac{\partial \theta}{\partial x_{j}}+\frac{\partial K_{\alpha \beta}^{\prime \prime}}{\partial x_{j}}
$$




$$
\begin{aligned}
\frac{\partial}{\partial x_{j}}\left(\rho D \frac{\partial K_{\alpha \beta}}{\partial x_{j}}\right) & =\frac{\partial}{\partial x_{j}}\left(\rho D \frac{\partial G_{\alpha \beta}}{\partial x_{j}}\right)+\frac{\partial G_{\alpha \beta}}{\partial \xi}\left(\rho D \frac{\partial \theta}{\partial x_{j}}\right) \\
& +\rho D \frac{\partial \theta}{\partial x_{j}} \frac{\partial \theta}{\partial x_{j}} \frac{\partial^{2} G_{\alpha \beta}}{\partial \xi^{2}}+\rho D \frac{\partial \theta}{\partial x_{j}} \frac{\partial}{\partial x_{j}} \frac{\partial G_{\alpha \beta}}{\partial \xi} \\
& +\frac{\partial}{\partial x_{j}}\left(\rho D \frac{\partial K_{\alpha \beta}^{\prime \prime}}{\partial x_{j}}\right) .
\end{aligned}
$$

By substituting Eq. (A.4) and (A.5) into Eq. (A.2) and rearranging the terms, the following equation is obtained:

$$
\begin{aligned}
\rho \frac{\partial G_{\alpha \beta}}{\partial t} & =-\rho v_{j} \frac{\partial G_{\alpha \beta}}{\partial x_{j}} \\
& +\frac{\partial}{\partial x_{j}}\left(\rho D \frac{\partial G_{\alpha \beta}}{\partial x_{j}}\right)+\rho D \frac{\partial \theta}{\partial x_{j}} \frac{\partial}{\partial x_{j}} \frac{\partial G_{\alpha \beta}}{\partial \xi} \\
& +\rho D \frac{\partial \theta}{\partial x_{j}} \frac{\partial \theta}{\partial x_{j}} \frac{\partial^{2} G_{\alpha \beta}}{\partial \xi^{2}} \\
& -\frac{\partial G_{\alpha \beta}}{\partial \xi}\left(\rho \frac{\partial \theta}{\partial t}+\rho v_{j} \frac{\partial \theta}{\partial x_{j}}-\frac{\partial}{\partial x_{j}}\left(\rho D \frac{\partial \theta}{\partial x_{j}}\right)\right) \\
& -2 \rho D\left(\frac{\partial Y_{\alpha}^{\prime \prime}}{\partial x_{j}} \frac{\partial Y_{\beta}^{\prime \prime}}{\partial x_{j}}\right) \\
& -\rho Y_{\beta}^{\prime \prime} \frac{\partial Q_{\alpha}}{\partial t}-\rho Y_{\alpha}^{\prime \prime} \frac{\partial Q_{\beta}}{\partial t} \\
& -\rho v_{j} Y_{\beta}^{\prime \prime} \frac{\partial Q_{\alpha}}{\partial x_{j}}-\rho v_{j} Y_{\alpha}^{\prime \prime} \frac{\partial Q_{\beta}}{\partial x_{j}} \\
& -\rho \frac{\partial K_{\alpha \beta}^{\prime \prime}}{\partial t}-\rho v_{j} \frac{\partial K_{\alpha \beta}^{\prime \prime}}{\partial x_{j}}+\frac{\partial}{\partial x_{j}}\left(\rho D \frac{\partial K_{\alpha \beta}^{\prime \prime}}{\partial x_{j}}\right) \\
& -\rho S_{\theta} Y_{\beta}^{\prime \prime} \frac{\partial Q_{\alpha}}{\partial \xi}-\rho S_{\theta} Y_{\alpha}^{\prime \prime} \frac{\partial Q_{\beta}}{\partial \xi} \\
& +\rho N Y_{\beta}^{\prime \prime} \frac{\partial^{2} Q_{\alpha}}{\partial \xi^{2}}+\rho N Y_{\alpha}^{\prime \prime} \frac{\partial^{2} Q_{\beta}}{\partial \xi^{2}} \\
& +Y_{\beta}^{\prime \prime} \frac{\partial}{\partial x_{j}}\left(\rho D \frac{\partial Q_{\alpha}}{\partial x_{j}}\right)+Y_{\alpha}^{\prime \prime} \frac{\partial}{\partial x_{j}}\left(\rho D \frac{\partial Q_{\beta}}{\partial x_{j}}\right) \\
& +\rho D Y_{\beta}^{\prime \prime} \frac{\partial \theta}{\partial x_{j}}\left(\frac{\partial}{\partial x_{j}} \frac{\partial Q_{\alpha}}{\partial \xi}\right)+\rho D Y_{\alpha}^{\prime \prime} \frac{\partial \theta}{\partial x_{j}}\left(\frac{\partial}{\partial x_{j}} \frac{\partial Q_{\beta}}{\partial \xi}\right) \\
& +\rho \dot{W}_{\alpha} Y_{\beta}^{\prime \prime}+\rho \dot{W}_{\beta} Y_{\alpha}^{\prime \prime} .
\end{aligned}
$$

Then by decomposing $v_{j}, N, S_{\theta}$ and $\dot{W}_{\alpha}$ to their conditional mean, $\left\langle\left.()\right|_{\xi}\right\rangle$, and fluctuations, ()$^{\prime \prime}$, and taking conditional average of the resulting equation leads to:

$$
\overline{\left.\rho\right|_{\xi}} \frac{\partial G_{\alpha \beta}}{\partial t}=-\overline{\left.\rho\right|_{\xi}}\left\langle\left. v_{j}\right|_{\xi}\right\rangle \frac{\partial G_{\alpha \beta}}{\partial x_{j}}
$$




$$
\begin{aligned}
& +\left\langle\left.\left(\frac{\partial}{\partial x_{j}}\left(\rho D \frac{\partial G_{\alpha \beta}}{\partial x_{j}}\right)+\rho D \frac{\partial \theta}{\partial x_{j}} \frac{\partial}{\partial x_{j}} \frac{\partial G_{\alpha \beta}}{\partial \xi}\right)\right|_{\xi}\right\rangle \\
& +\overline{\left.\rho\right|_{\xi}}\left\langle\left. N\right|_{\xi}\right\rangle \frac{\partial^{2} G_{\alpha \beta}}{\partial \xi^{2}}-\overline{\left.\rho\right|_{\xi}}\left\langle\left. S_{\theta}\right|_{\xi}\right\rangle \frac{\partial G_{\alpha \beta}}{\partial \xi} \\
& -2 \overline{\left.\rho\right|_{\xi}}\left\langle\left. D \frac{\partial Y_{\alpha}^{\prime \prime}}{\partial x_{j}} \frac{\partial Y_{\beta}^{\prime \prime}}{\partial x_{j}}\right|_{\xi}\right\rangle \\
& -\left\langle\left.\left(\rho \frac{\partial K_{\alpha \beta}^{\prime \prime}}{\partial t}+\rho v_{j} \frac{\partial K_{\alpha \beta}^{\prime \prime}}{\partial x_{j}}-\frac{\partial}{\partial x_{j}}\left(\rho D \frac{\partial K_{\alpha \beta}^{\prime \prime}}{\partial x_{j}}\right)\right)\right|_{\xi}\right\rangle \\
& -\overline{\left.\rho\right|_{\xi}}\left\langle\left. v_{j}^{\prime \prime} Y_{\beta}^{\prime \prime}\right|_{\xi}\right\rangle \frac{\partial Q_{\alpha}}{\partial x_{j}}-\overline{\left.\rho\right|_{\xi}}\left\langle\left. v_{j}^{\prime \prime} Y_{\alpha}^{\prime \prime}\right|_{\xi}\right\rangle \frac{\partial Q_{\beta}}{\partial x_{j}} \\
& -\overline{\left.\rho\right|_{\xi}}\left\langle\left. S_{\theta}^{\prime \prime} Y_{\beta}^{\prime \prime}\right|_{\xi}\right\rangle \frac{\partial Q_{\alpha}}{\partial \xi}-\overline{\left.\rho\right|_{\xi}}\left\langle\left. S_{\theta}^{\prime \prime} Y_{\alpha}^{\prime \prime}\right|_{\xi}\right\rangle \frac{\partial Q_{\beta}}{\partial \xi} \\
& +\overline{\left.\rho\right|_{\xi}}\left\langle\left. N^{\prime \prime} Y_{\beta}^{\prime \prime}\right|_{\xi}\right\rangle \frac{\partial^{2} Q_{\alpha}}{\partial \xi^{2}}+\overline{\left.\rho\right|_{\xi}}\left\langle\left. N^{\prime \prime} Y_{\alpha}^{\prime \prime}\right|_{\xi}\right\rangle \frac{\partial^{2} Q_{\beta}}{\partial \xi^{2}} \\
& +\left\langle\left.\left(Y_{\beta}^{\prime \prime} \frac{\partial}{\partial x_{j}}\left(\rho D \frac{\partial Q_{\alpha}}{\partial x_{j}}\right)+Y_{\alpha}^{\prime \prime} \frac{\partial}{\partial x_{j}}\left(\rho D \frac{\partial Q_{\beta}}{\partial x_{j}}\right)\right)\right|_{\xi}\right\rangle \\
& +\left\langle\left.\left(\rho D Y_{\beta}^{\prime \prime} \frac{\partial \theta}{\partial x_{j}}\left(\frac{\partial}{\partial x_{j}} \frac{\partial Q_{\alpha}}{\partial \xi}\right)+\rho D Y_{\alpha}^{\prime \prime} \frac{\partial \theta}{\partial x_{j}}\left(\frac{\partial}{\partial x_{j}} \frac{\partial Q_{\beta}}{\partial \xi}\right)\right)\right|_{\xi}\right\rangle \\
& +\overline{\left.\rho\right|_{\xi}}\left\langle\left.\dot{W}_{\alpha}^{\prime \prime} Y_{\beta}^{\prime \prime}\right|_{\xi}\right\rangle+\overline{\left.\rho\right|_{\xi}}\left\langle\left.\dot{W}_{\beta}^{\prime \prime} Y_{\alpha}^{\prime \prime}\right|_{\xi}\right\rangle .
\end{aligned}
$$

By assuming that the cylinder charge is statistically homogeneous, all spatial derivatives can be neglected and the variance equation can be obtained as:

$$
\begin{aligned}
\frac{\partial G_{\alpha \alpha}}{\partial t} & =\left\langle\left. N\right|_{\xi}\right\rangle \frac{\partial^{2} G_{\alpha \alpha}}{\partial \xi^{2}}-\left\langle\left. S_{\theta}\right|_{\xi}\right\rangle \frac{\partial G_{\alpha \alpha}}{\partial \xi} \\
& +2\left\langle\left.\dot{W}_{\alpha}^{\prime \prime} Y_{\alpha}^{\prime \prime}\right|_{\xi}\right\rangle+2\left\langle\left. N^{\prime \prime} Y_{\alpha}^{\prime \prime}\right|_{\xi}\right\rangle \frac{\partial^{2} Q_{\alpha}}{\partial \xi^{2}}-2\left\langle\left. S_{\theta}^{\prime \prime} Y_{\alpha}^{\prime \prime}\right|_{\xi}\right\rangle \frac{\partial Q_{\alpha}}{\partial \xi} .
\end{aligned}
$$

\section{References}

1. Dec, J.E.: Advanced compression-ignition engines-understanding the in-cylinder processes. Proc. Combust. Inst. 32(2), 2727-2742 (2009)

2. Premier, A.: HCCI could cut fuel consumption by 15\%. Adv Mater Process. p. 27 (2007)

3. Dec, J.E., Hwang, W., Sjöberg, M.: An investigation of thermal stratification in HCCI engines using chemiluminescence imaging. SAE Paper 2006-01-1518 (2006)

4. Liu, H., Yao, M., Zheng, Z., Wang, Y.: An investigation of different ported fuel injection strategies and thermal stratification in HCCI engines using chemiluminescence imaging. SAE Paper 2010-01-0163 (2010)

5. Snyder, J., Dronniou, N., Dec, J., Hanson, R.: PLIF measurements of thermal stratification in an HCCI engine under fired operation. SAE Int. J. Engines 5(1), 1669-1688 (2011)

6. Yang, Y., Dec, J.E., Dronniou, N., Sjöberg, M.: Tailoring HCCI heat-release rates with partial fuel stratification: Comparison of two-stage and single-stage-ignition fuels. Proc. Combust. Inst. 33(2), 3047-3055 (2011) 
7. Krasselt, J., Foster, D., Ghandhi, J., Herold, R., Reuss, D., Najt, P.: Investigations into the effects of thermal and compositional stratification on HCCI combustion part I: Metal engine results. SAE Paper 2009-01-1105 (2009)

8. Chen, J.H., Hawkes, E.R., Sankaran, R., Mason, S.D., Im, H.G.: Direct numerical simulation of ignition front propagation in a constant volume with temperature inhomogeneities: I. Fundamental analysis and diagnostics. Combust. Flame 145(12), 128-144 (2006)

9. Hawkes, E.R., Sankaran, R., Pébay, P.P., Chen, J.H.: Direct numerical simulation of ignition front propagation in a constant volume with temperature inhomogeneities: II. Parametric study. Combust. Flame 145(12), 145-159 (2006)

10. Bansal, G., Im, H.G.: Autoignition and front propagation in low temperature combustion engine environments. Combust. Flame 158(11), 2105-2112 (2011)

11. Yu, R., Bai, X.S.: Direct numerical simulation of lean hydrogen/air auto-ignition in a constant volume enclosure. Combust. Flame 160(9), 1706 - 1716 (2013)

12. Talei, M., Hawkes, E.R.: Ignition in compositionally and thermally stratified $n$ heptane/air mixtures: A direct numerical simulation study. Proc. Combust. Inst. 35(3), 3027-3035 (2015)

13. Yoo, C.S., Lu, T., Chen, J.H., Law, C.K.: Direct numerical simulations of ignition of a lean $n$-heptane/air mixture with temperature inhomogeneities at constant volume: Parametric study. Combust. Flame 158(9), 1727-1741 (2011)

14. Yoo, C.S., Luo, Z., Lu, T., Kim, H., Chen, J.H.: A DNS study of ignition characteristics of a lean iso-octane/air mixture under HCCI and SACI conditions. Proc. Combust. Inst. 34(2), 2985-2993 (2013)

15. Zhang, H., Hawkes, E.R., Chen, J.H., Kook, S.: A numerical study of the autoignition of dimethyl ether with temperature inhomogeneities. Proc. Combust. Inst. 34(1), 803-812 (2013)

16. El-Asrag, H.A., Ju, Y.: Direct numerical simulations of exhaust gas recirculation effect on multistage autoignition in the negative temperature combustion regime for stratified $\mathrm{HCCI}$ flow conditions by using $\mathrm{H}_{2} \mathrm{O}_{2}$ addition. Combust. Theor. Model. 17(2), 316-334 (2013)

17. Aceves, S.M., Flowers, D.L., Espinosa-Loza, F., Martinez-Frias, J., Dec, J.E., Sjöberg, M., Dibble, R.W., Hessel, R.P.: Spatial analysis of emissions sources for HCCI combustion at low loads using a multi-zone model. SAE Paper 2004-01-1910 (2004)

18. Krisman, A., Hawkes, E.R., Kook, S., Sjöberg, M., Dec, J.E.: On the potential of ethanol fuel stratification to extend the high load limit in stratified-charge compression-ignition engines. Fuel 99(0), 45-54 (2012)

19. Cook, D.J., Pitsch, H., Chen, J.H., Hawkes, E.R.: Flamelet-based modeling of autoignition with thermal inhomogeneities for application to HCCI engines. Proc. Combust. Inst. 31(2), 2903-2911 (2007)

20. Hu, B., Rutland, C.J., Shethaji, T.A.: Combustion modeling of conventional diesel-type and HCCI-type diesel combustion with large eddy simulations. SAE Paper 2008-01-0958 (2008)

21. Cook, D.J., Pitsch, H.: Enthalpy-based flamelet model for HCCI applied to a rapid compression machine. SAE Paper 2005-01-3735 (2005)

22. Cook, D.J., Pitsch, H., Nentwig, G.: Numerical investigation of unburnt hydrocarbon emissions in a homogeneous-charge late-injection diesel-fueled engine. SAE Paper 200801-1666 (2008)

23. Dahms, R., Felsch, C., Röhl, O., Peters, N.: Detailed chemistry flamelet modeling of mixed-mode combustion in spark-assisted HCCI engines. Proc. Combust. Inst. 33(2), $3023-3030$ (2011)

24. Colin, O., da Cruz, A.P., Jay, S.: Detailed chemistry-based auto-ignition model including low temperature phenomena applied to 3-D engine calculations. Proc. Combust. Inst. 30(2), 2649-2656 (2005)

25. Pera, C., Colin, O., Jay, S.: Development of a FPI detailed chemistry tabulation methodology for internal combustion engines. Oil \& Gas Science and Technology - Rev. IFP 64(3), 243-258 (2009)

26. Jay, S., Colin, O.: Modeling of pollutant emissions using combined tabulated detailed kinetics and reduced kinetics. SAE Paper 2010-01-0628 (2010) 
27. Bisetti, F., Chen, J.Y., Hawkes, E.R., Chen, J.H.: Probability density function treatment of turbulence chemistry interactions during the ignition of a temperature-stratified mixture for application to HCCI engine modeling. Combust. Flame 155(4), 571-584 (2008)

28. Zhang, Y., Kung, E., Haworth, D.: A PDF method for multidimensional modeling of HCCI engine combustion: effects of turbulence/chemistry interactions on ignition timing and emissions. Proc. Combust. Inst. 30(2), 2763-2771 (2005)

29. Salehi, F., Talei, M., Hawkes, E.R., Yoo, C.S., Lucchini, T., D’Errico, G., Kook, S.: Conditional moment closure modelling for HCCI with temperature inhomogeneities. Proc. Combust. Inst. 35(3), 3087-3095 (2015)

30. Klimenko, A.Y.: Multicomponent diffusion of various admixtures in turbulent flow. Fluid Dyn. 25(3), 327-334 (1990)

31. Bilger, R.: Conditional moment closure for turbulent reacting flow. Phys. Fluids A 5(2), 436-444 (1993)

32. Navarro-Martinez, S., Kronenburg, A.: LES-CMC simulations of a turbulent bluff-body flame. Proc. Combust. Inst. 31(2), 1721-1728 (2007)

33. Kim, G., Kang, S., Kim, Y., Bilger, R.W., Cleary, M.J.: Conditional moment closure and transient flamelet modelling for detailed structure and $\mathrm{NO}_{\mathrm{x}}$ formation characteristics of turbulent nonpremixed jet and recirculating flames. Combust. Theor. Model. 11(4), $527-552(2007)$

34. Richardson, E., Mastorakos, E.: Conditional moment closure modelling for spark ignition in a turbulent $n$-heptane spray. 5th Mediterranean combustion symposium (2007)

35. Navarro-Martinez, S., Kronenburg, A.: LES-CMC simulations of a lifted methane flame. Proc. Combust. Inst. 32(1), 1509-1516 (2009)

36. Devaud, C., Bray, K.: Assessment of the applicability of conditional moment closure to a lifted turbulent flame: first order model. Combust. Flame 132(12), $102-114$ (2003)

37. El Sayed, A., Devaud, C.B.: Conditional moment closure (CMC) applied to autoignition of high pressure methane jets in a shock tube. Combust. Theor. Model. 12(5), 943-972 (2008)

38. Buckrell, A., Devaud, C.: Investigation of mixing models and conditional moment closure applied to autoignition of hydrogen jets. Flow Turbul. Combust. 90(3), 621-644 (2013)

39. Cleary, M.J., Kent, J.H., Bilger, R.W.: Prediction of carbon monoxide in fires by conditional moment closure. Proc. Combust. Inst. 29(1), 273-279 (2002)

40. Cleary, M., Kent, J.: Modelling of species in hood fires by conditional moment closure. Combust. Flame 143(4), 357-368 (2005)

41. Wright, Y.M., Paola, G.D., Boulouchos, K., Mastorakos, E.: Simulations of spray autoignition and flame establishment with two-dimensional CMC. Combust. Flame 143(4), 402-419 (2005)

42. De Paola, G., Mastorakos, E., Wright, Y.M., Boulouchos, K.: Diesel engine simulations with multi-dimensional conditional moment closure. Combustion Science and Technology 180(5), 883-899 (2008)

43. Wright, Y.M., Boulouchos, K., Paola, G.D., Mastorakos, E.: Multi-dimensional conditional moment closure modelling applied to a heavy-duty common-rail diesel engine. SAE Int. J. Engines 2, 714-726 (2009)

44. Seo, J., Lee, D., Huh, K.Y., Chung, J.: Combustion simulation of a diesel engine in the pHCCI mode with split injections by the spatially integrated CMC model. Combust. Sci. Tech. 182(9), 1241-1260 (2010)

45. Borghesi, G., Mastorakos, E., Devaud, C.B., Bilger, R.W.: Modeling evaporation effects in conditional moment closure for spray autoignition. Combust. Theor. Model. 15(5), 725-752 (2011)

46. Bolla, M., Wright, Y.M., Boulouchos, K., Borghesi, G., Mastorakos, E.: Soot formation modeling of $n$-heptane sprays under diesel engine conditions using the conditional moment closure approach. Combust. Sci. Tech. 185(5), 766-793 (2013)

47. Swaminathan, N., Bilger, R.W.: Analyses of conditional moment closure for turbulent premixed flames. Combust. Theor. Model. 5(2), 241-260 (2001)

48. Amzin, S., Swaminathan, N., Rogerson, J.W., Kent, J.H.: Conditional moment closure for turbulent premixed flames. Combust. Sci. Tech. 184(10-11), 1743-1767 (2012) 
49. Thornber, B., Bilger, R.W., Masri, A.R., Hawkes, E.R.: An algorithm for LES of premixed compressible flows using the conditional moment closure model. J. Comput. Phys. 230(20), 7687-7705 (2011)

50. Klimenko, A.Y., Bilger, R.W.: Conditional moment closure for turbulent combustion. Prog. Energ. Combust. Sci. 25(6), 595-687 (1999)

51. Zeldovich, Y.B.: Regime classification of an exothermic reaction with nonuniform initial conditions. Combust. Flame 39(2), 211-214 (1980)

52. Klimenko, A.: On the relation between the conditional moment closure and unsteady flamelets. Combust. Theor. Model. 5(3), 275-294 (2001)

53. Sankaran, R., Hawkes, E.R., Chen, J.H., Lu, T., Law, C.K.: Direct numerical simulations of turbulent lean premixed combustion. J. Phys. Conf. Ser. 46(1), 38 (2006)

54. Hawkes, E.R., Sankaran, R., Chen, J.H., Kaiser, S.A., Frank, J.H.: An analysis of lowerdimensional approximations to the scalar dissipation rate using direct numerical simulations of plane jet flames. Proc. Combust. Inst. 32(1), 1455-1463 (2009)

55. Hawkes, E.R., Sankaran, R., Chen, J.H.: Estimates of the three-dimensional flame surface density and every term in its transport equation from two-dimensional measurements. Proc. Combust. Inst. 33(1), 1447-1454 (2011)

56. Chen, J.H.: Petascale direct numerical simulation of turbulent combustion-fundamental insights towards predictive models. Proc. Combust. Inst. 33(1), 99-123 (2011)

57. Gruber, A., Chen, J.H., Valiev, D., Law, C.K.: Direct numerical simulation of premixed flame boundary layer flashback in turbulent channel flow. J. Fluid Mech. 709, 516-542 (2012)

58. Kolla, H., Grout, R.W., Gruber, A., Chen, J.H.: Mechanisms of flame stabilization and blowout in a reacting turbulent hydrogen jet in cross-flow. Combust. Flame 159(8), 2755-2766 (2012)

59. Chatakonda, O., Hawkes, E.R., Aspden, A.J., Kerstein, A.R., Kolla, H., Chen, J.H.: On the fractal characteristics of low Damköhler number flames. Combust. Flame 160(11), 242-2433 (2013)

60. Pope, S.: Turbulent Flowes. Cambridge (2000)

61. Kronenburg, A., Bilger, R., Kent, J.: Second-order conditional moment closure for turbulent jet diffusion flames. Proc. Combust. Inst. 27(1), 1097-1104 (1998)

62. Swaminathan, N., Bilger, R.W.: Study of the conditional covariance and variance equations for second order conditional moment closure. Phys. Fluids 11(9), 2679-2695 (1999)

63. Kim, S.H., Huh, K.Y.: Second-order conditional moment closure modeling of turbulent piloted jet diffusion flames. Combust. Flame 138(4), 336-352 (2004)

64. Richardson, E., Yoo, C., Chen, J.: Analysis of second-order conditional moment closure applied to an autoignitive lifted hydrogen jet flame. Proc. Combust. Inst. 32(2), 16951703 (2009)

65. Paola, G., Kim, I., Mastorakos, E.: Second-order conditional moment closure simulations of autoignition of an $n$-heptane plume in a turbulent coflow of heated air. Flow Turbul. Combust. 82(4), 455-475 (2009) 


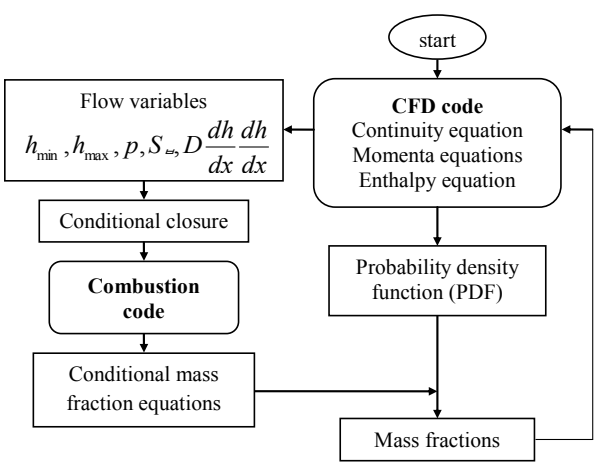

Fig. 1 Schematic diagram of the solution algorithm.
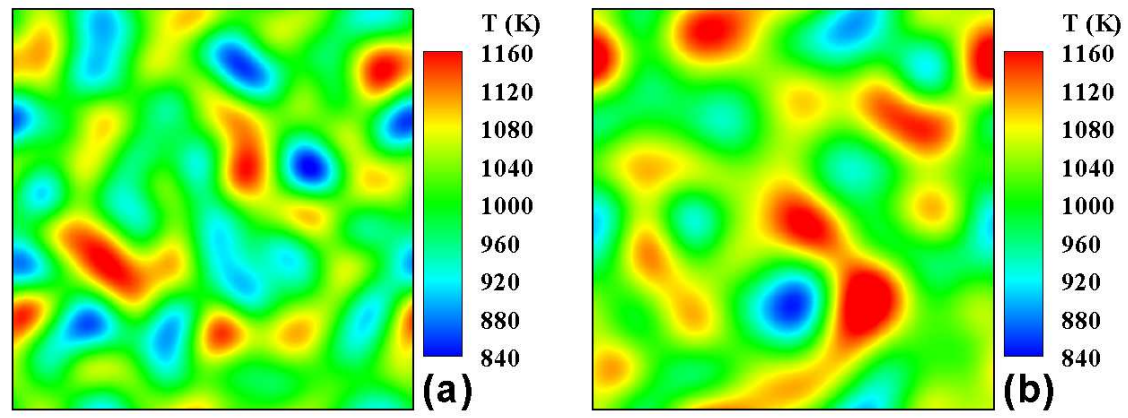

Fig. 2 Initial temperature for case a) heptane- 12 with $T^{\prime}=60 \mathrm{~K}$ and b) octane- 3 with $T^{\prime}=60 \mathrm{~K}$. 
Table 1 Physical parameters of the DNS and CMC cases.

\begin{tabular}{ccccc}
\hline Case & $T_{0}(\mathrm{~K})$ & $T^{\prime}(\mathrm{K})$ & $u^{\prime}(\mathrm{m} / \mathrm{s})$ & $l_{e}(\mathrm{~mm})$ \\
\hline octane-1 & 1035 & 15 & 0.5 & 1.25 \\
octane-2 & 1035 & 30 & 0.5 & 1.25 \\
octane-3 & 1035 & 60 & 0.5 & 1.25 \\
octane-8 & 1035 & 15 & 2.5 & 1.25 \\
octane-9 & 1035 & 15 & 0.5 & 0.25 \\
heptane-10 & 1008 & 15 & 0.5 & 1.24 \\
heptane-11 & 1008 & 30 & 0.5 & 1.24 \\
heptane-12 & 1008 & 60 & 0.5 & 1.24 \\
\hline
\end{tabular}
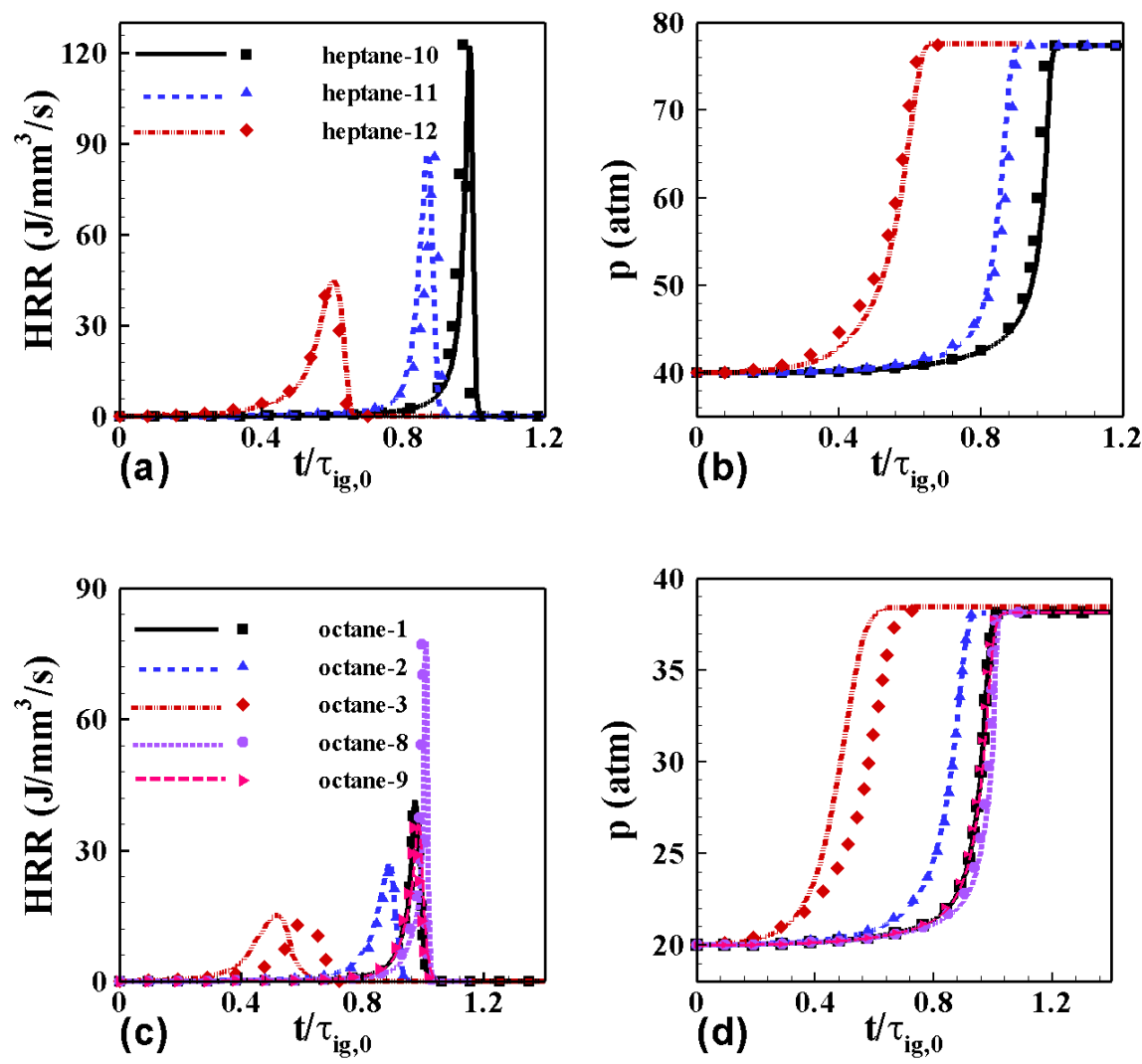

Fig. 3 Temporal evolution of the a) mean heat release rate and b) pressure obtained using CFD-CMC solver (lines) in comparison with DNS (symbols) for a-b) $n$-heptane and c-d) iso-octane cases. 


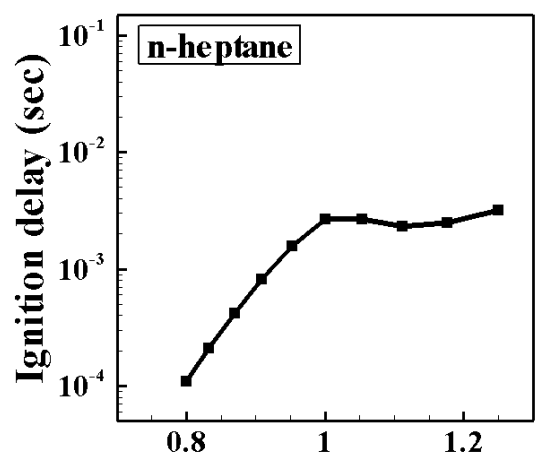

(a) $1000 / \mathrm{T}(1 / \mathrm{K})$

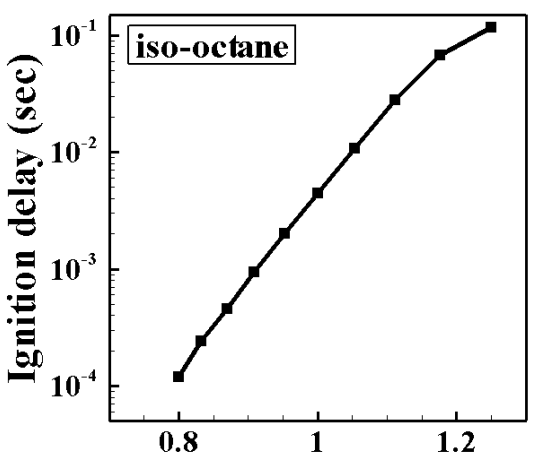

(b) $1000 / \mathrm{T}(1 / \mathrm{K})$

Fig. 4 Homogeneous ignition delay as a function of inverse of the temperature for a) $n$ heptane/air b) iso-octane/air mixture.
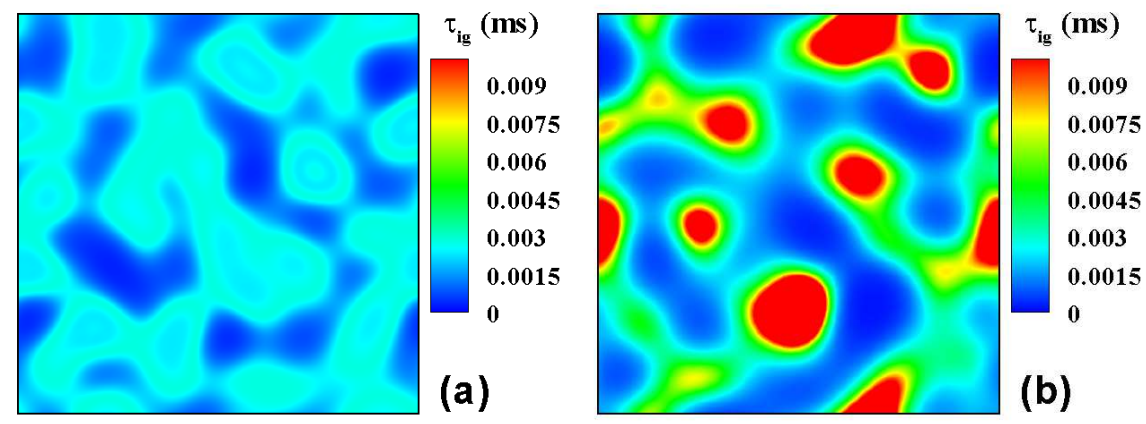

Fig. 5 Initial ignition delay time distribution for case a) heptane- 12 with $T^{\prime}=60 \mathrm{~K}$ and b) octane- 3 with $T^{\prime}=60 \mathrm{~K}$.

Table 2 Mean values predicted by CMC model compared to DNS results.

\begin{tabular}{cccccccccc}
\hline & \multicolumn{3}{c}{ DNS } & \multicolumn{3}{c}{ CMC } & \multicolumn{2}{c}{ Error } \\
\cline { 2 - 10 } Case & $\begin{array}{c}\tau_{i g}^{\prime} \\
(\mathrm{ms})\end{array}$ & $\begin{array}{c}\tau_{i g} \\
(\mathrm{~ms})\end{array}$ & $\begin{array}{c}\tau_{b} \\
(\mathrm{~ms})\end{array}$ & $\begin{array}{c}\mathrm{HRR}_{\max } \\
\left(\mathrm{J} / \mathrm{mm}^{3} / \mathrm{s}\right)\end{array}$ & $\begin{array}{c}\tau_{i g} \\
(\mathrm{~ms})\end{array}$ & $\begin{array}{c}\tau_{b} \\
(\mathrm{~ms})\end{array}$ & $\begin{array}{c}\mathrm{HRR}_{\max } \\
\left(\mathrm{J} / \mathrm{mm}^{3} / \mathrm{s}\right)\end{array}$ & $\begin{array}{c}\tau_{i g} \\
\%\end{array}$ & $\begin{array}{c}\text { HRR } \\
\%\end{array}$ \\
\hline octane-1 & 0.62 & 2.43 & 0.10 & 45.0 & 2.43 & 0.10 & 41.6 & 0.00 & 1.10 \\
octane-2 & 1.52 & 2.23 & 0.17 & 26.8 & 2.23 & 0.17 & 27.5 & 0.00 & 4.51 \\
octane-3 & 6.25 & 1.51 & 0.33 & 13.4 & 1.32 & 0.33 & 14.7 & 12.6 & 9.70 \\
octane-8 & 0.62 & 2.49 & 0.04 & 77.2 & 2.52 & 0.04 & 78.0 & 1.20 & 0.64 \\
octane-9 & 0.62 & 2.43 & 0.11 & 35.7 & 2.46 & 0.12 & 36.4 & 1.23 & 1.96 \\
heptane-10 & 0.24 & 2.43 & 0.07 & 123 & 2.47 & 0.07 & 123 & 1.65 & 0.10 \\
heptane-11 & 0.46 & 2.23 & 0.10 & 86.0 & 2.18 & 0.09 & 86.9 & 2.24 & 1.05 \\
heptane-12 & 0.69 & 1.50 & 0.19 & 45.4 & 1.52 & 0.20 & 47.5 & 1.33 & 4.63 \\
\hline
\end{tabular}



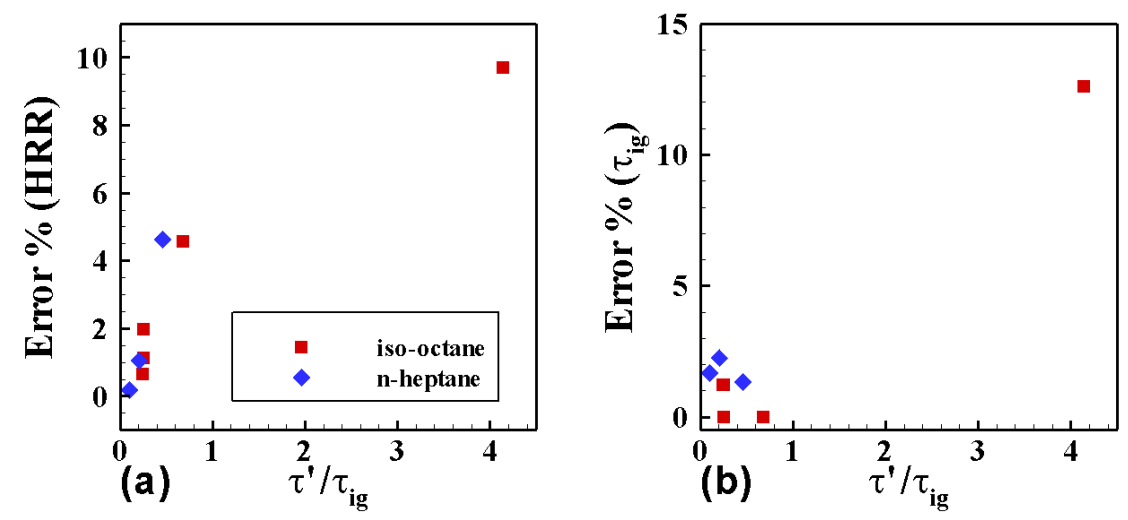

Fig. 6 Percentages of error for prediction of a) maximum of mean heat rate and b) ignition delay time for all $n$-heptane and iso-octane cases
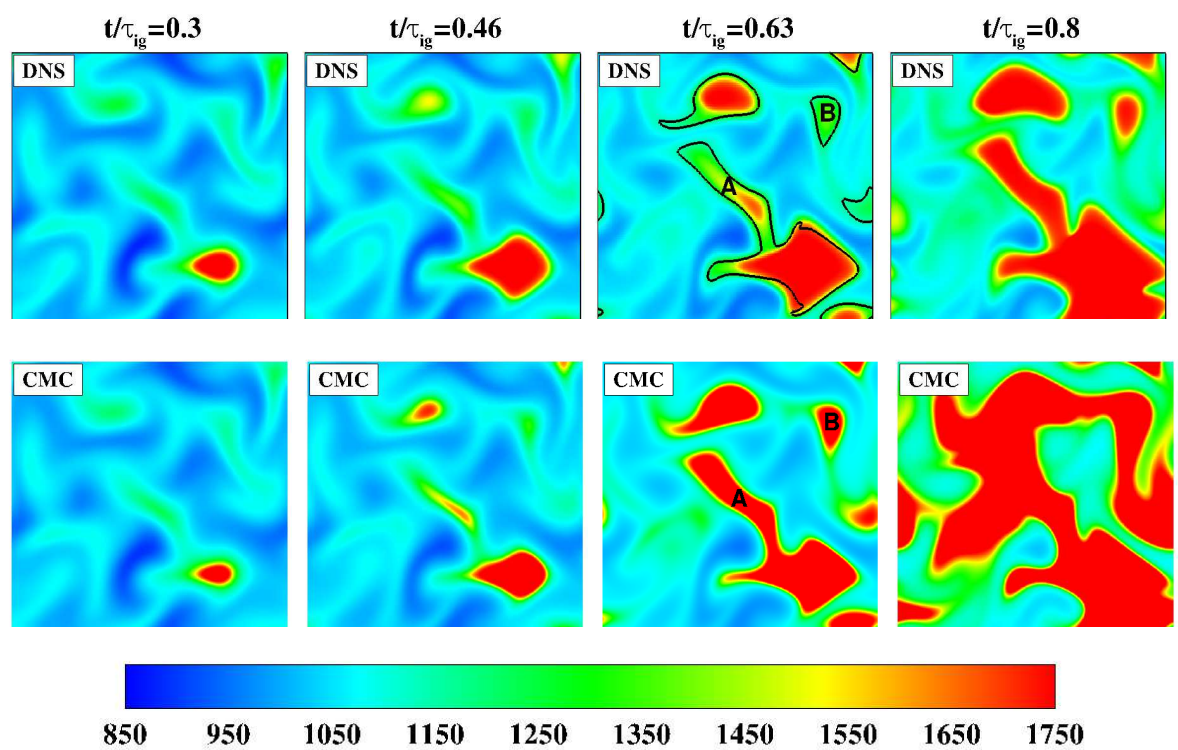

Fig. 7 Isocontours of temperature for case octane- 3 with $T^{\prime}=60 \mathrm{~K}$ obtained using DNS (above row) and CFD-CMC solver (bottom row) at $t / \tau_{i g}=0.3,0.46,0.63$ and 0.8 . 

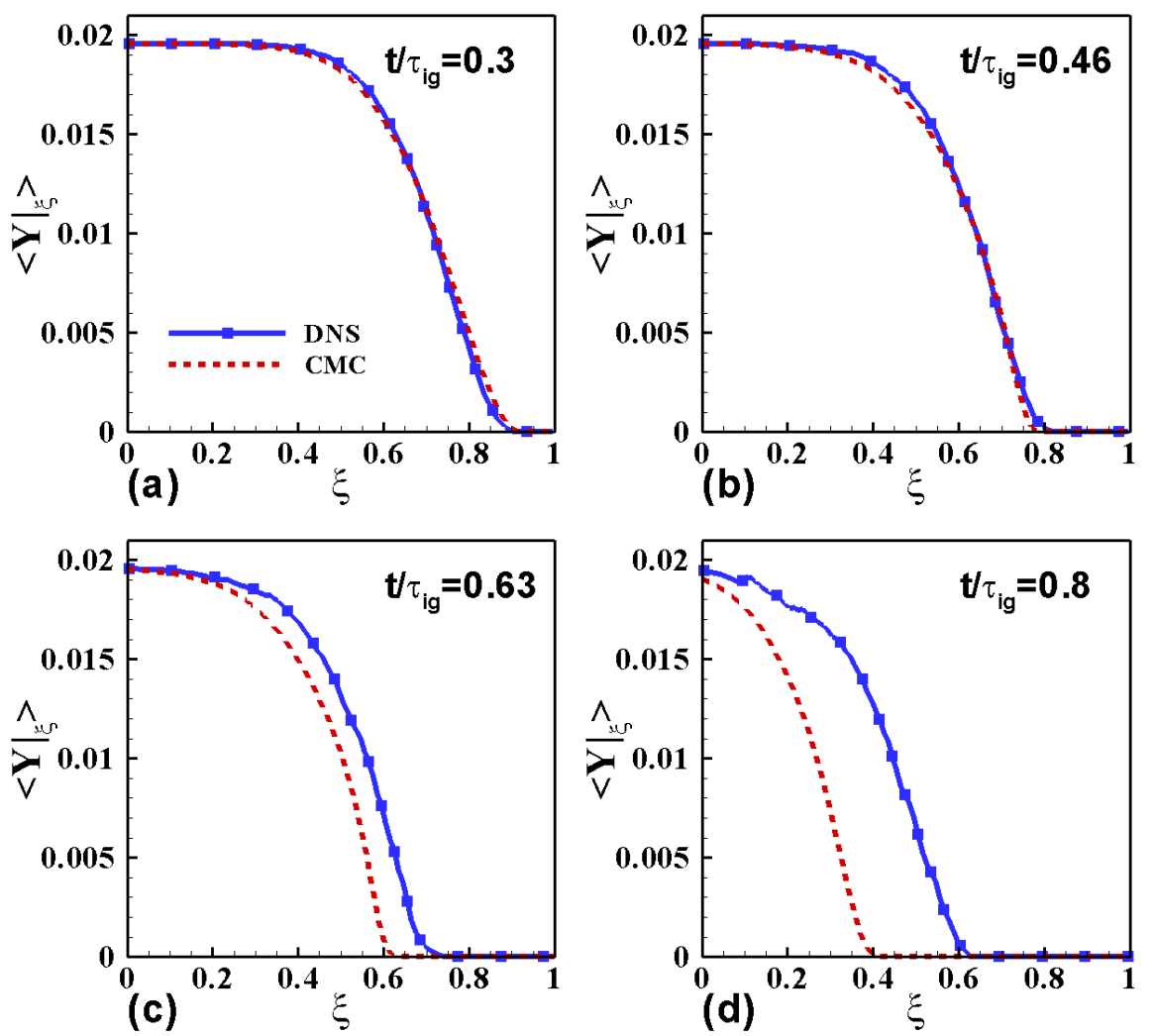

Fig. 8 Conditional mass fraction of fuel for case octane- 3 with $T^{\prime}=60 \mathrm{~K}$ obtained using DNS (blue solid line) and CFD-CMC solver (red dashed line) at $t / \tau_{i g}=0.3,0.46,0.63$ and 0.8 . 

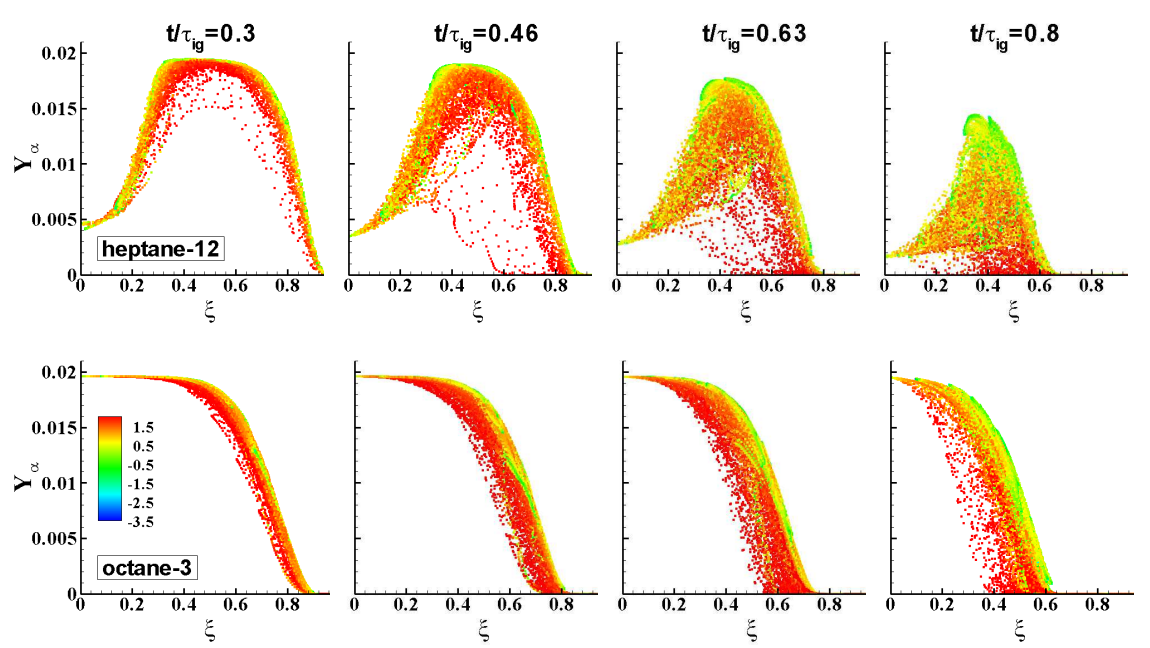

Fig. 9 Scatter-plot of fuel mass fraction versus normalised enthalpy for case octane-3 with $T^{\prime}=60 \mathrm{~K}$ (above row) and case heptane- 12 with $T^{\prime}=60 \mathrm{~K}$ (bottom row). 

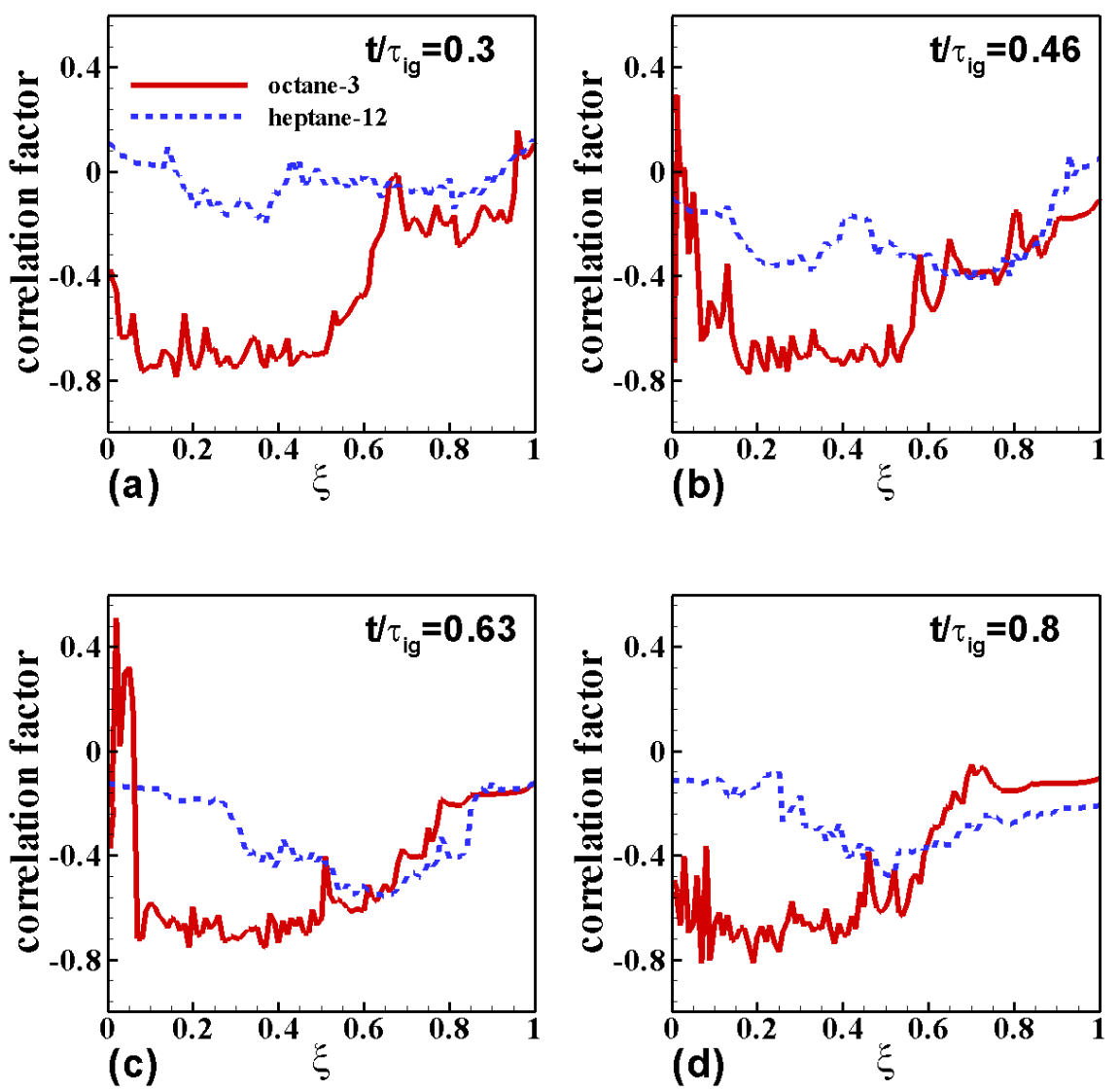

Fig. 10 Correlation factor between fuel mass fraction and dissipation rate at $t / \tau_{i g}=0.3$, $0.46,0.63$ and 0.8 for case octane-3 (red solid line) and case heptane-12 (dashed blue line). 


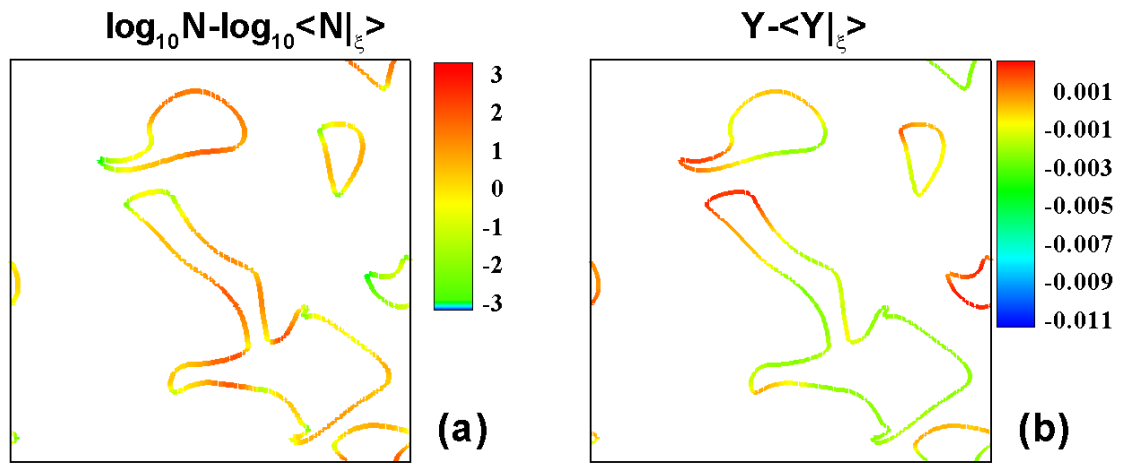

Fig. 11 Conditional a) dissipation rate fluctuations, $\log _{10} N-\log _{10}\langle N \mid \xi\rangle$, and b) mass fraction fluctuations, $Y_{\alpha}-\left\langle\left. Y_{\alpha}\right|_{\xi}\right\rangle$ along isocontour of $\xi=0.66$, at $t / \tau_{i g}=0.63$ for case octane-3 with $T^{\prime}=60 \mathrm{~K}$
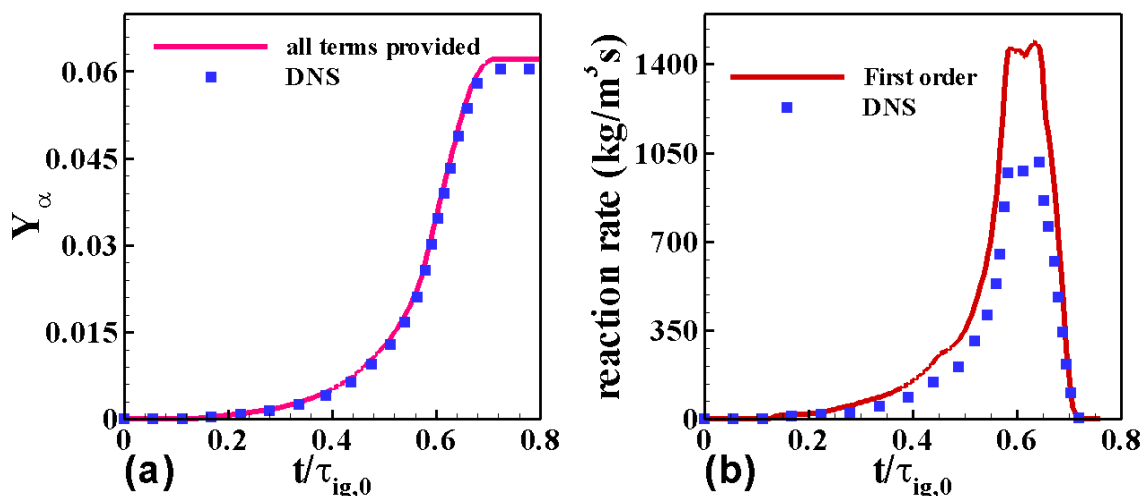

Fig. 12 Evaluation of first order assumption; temporal evolution of mean $\mathrm{CO}_{2}$ a) mass fraction and b) reaction rate for case octane- 3 with $T^{\prime}=60 \mathrm{~K}$. 

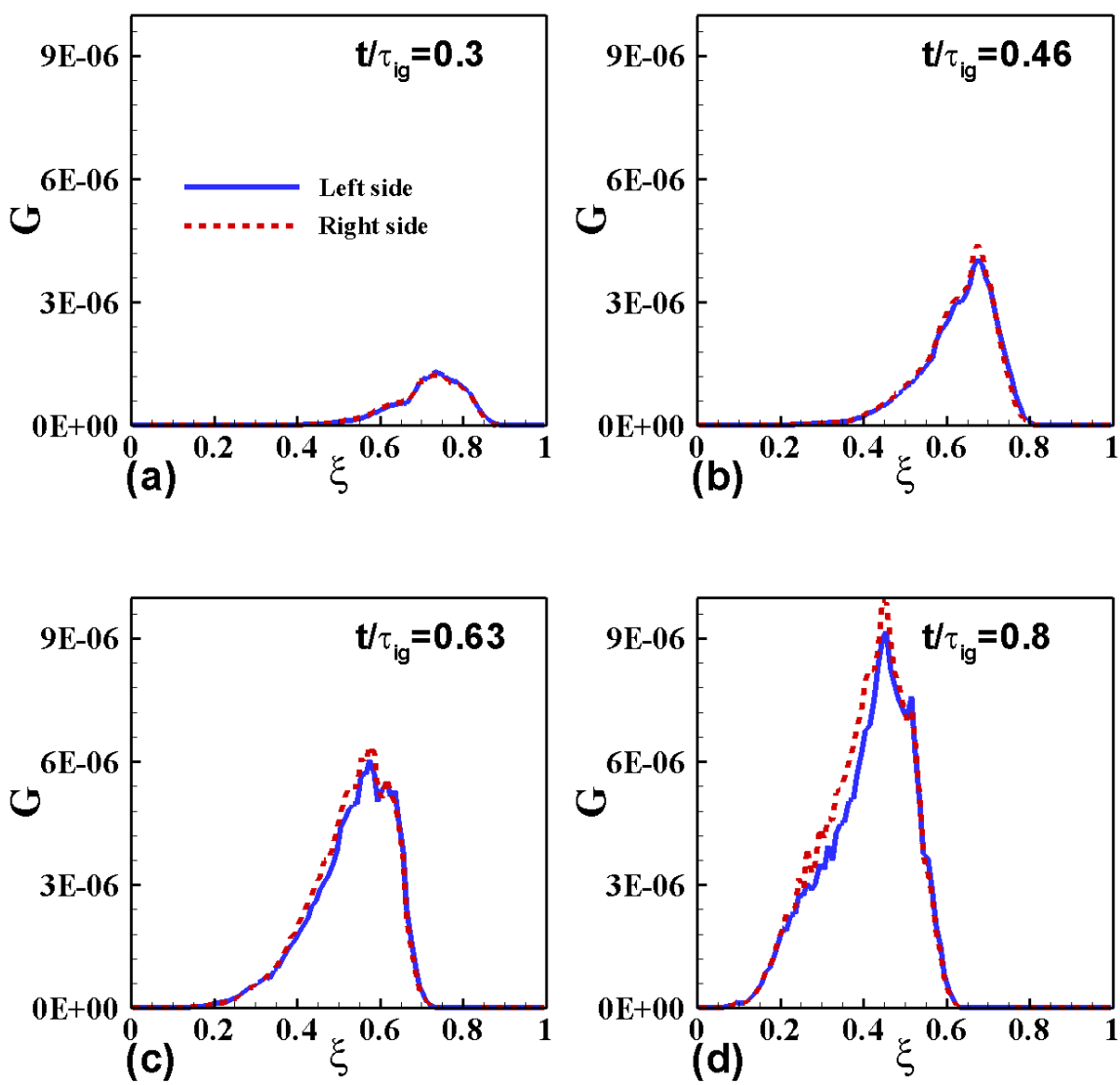

Fig. 13 Comparison of the conditional variance for fuel obtained using the integration of the right and left hand sides of Eq. (8) for case octane- 3 with $T^{\prime}=60 \mathrm{~K}$ at $t / \tau_{i g}=0.3$, $0.46,0.63$ and 0.8 . 

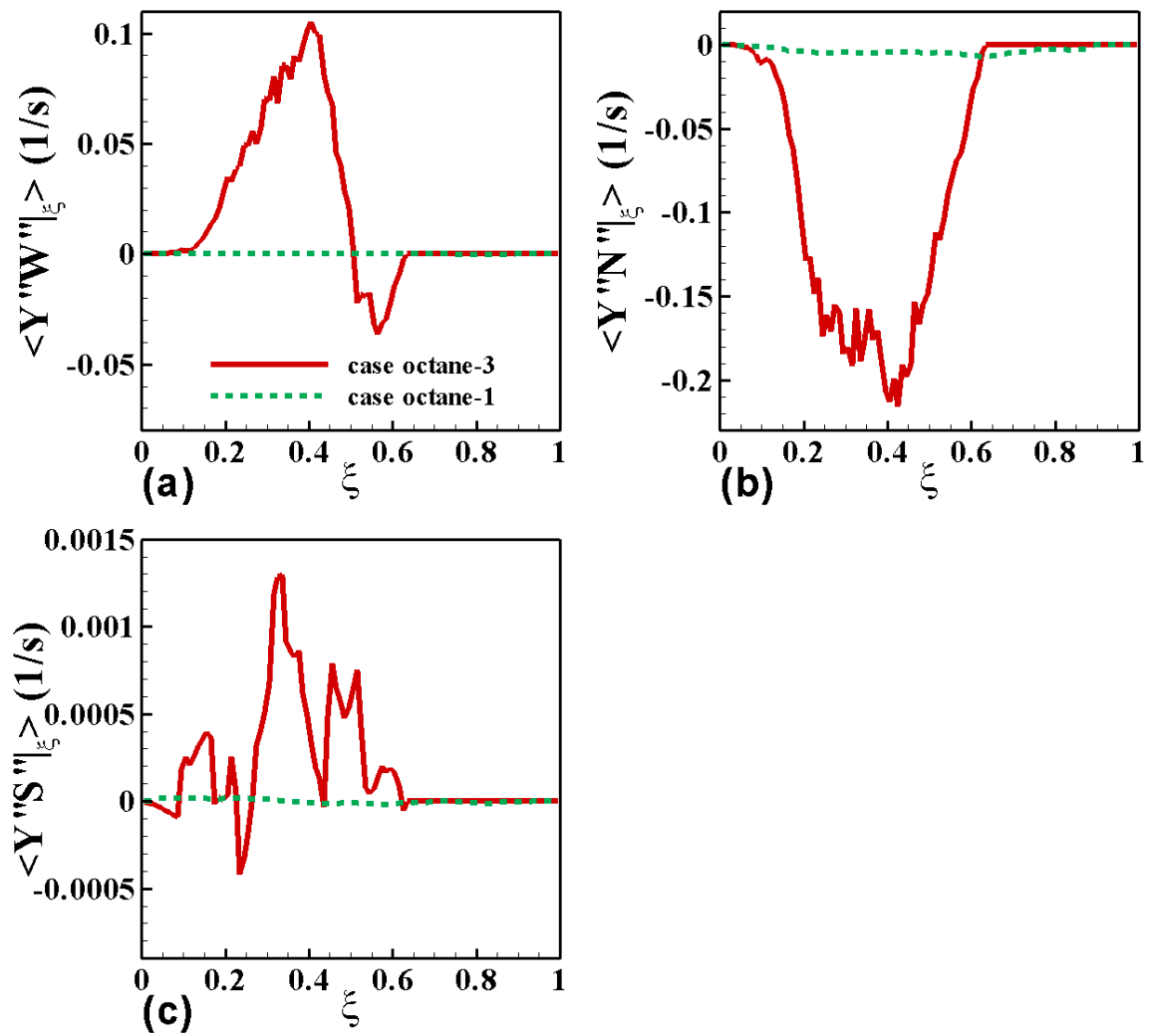

Fig. 14 Source terms in Eq. (8) including a) $\left\langle\left.\dot{W}_{\alpha}^{\prime \prime} Y_{\alpha}^{\prime \prime}\right|_{\xi}\right\rangle$, b) $\left\langle\left. N^{\prime \prime} Y_{\alpha}^{\prime \prime}\right|_{\xi}\right\rangle$ and c) $\left\langle\left. S_{\theta}^{\prime \prime} Y_{\alpha}^{\prime \prime}\right|_{\xi}\right\rangle$ for cases octane-1 with $T^{\prime}=15 \mathrm{~K}$ (dashed green line) and octane-3 with $T^{\prime}=60 \mathrm{~K}$ (red solid line) at $t / \tau_{i g}=0.8$. 

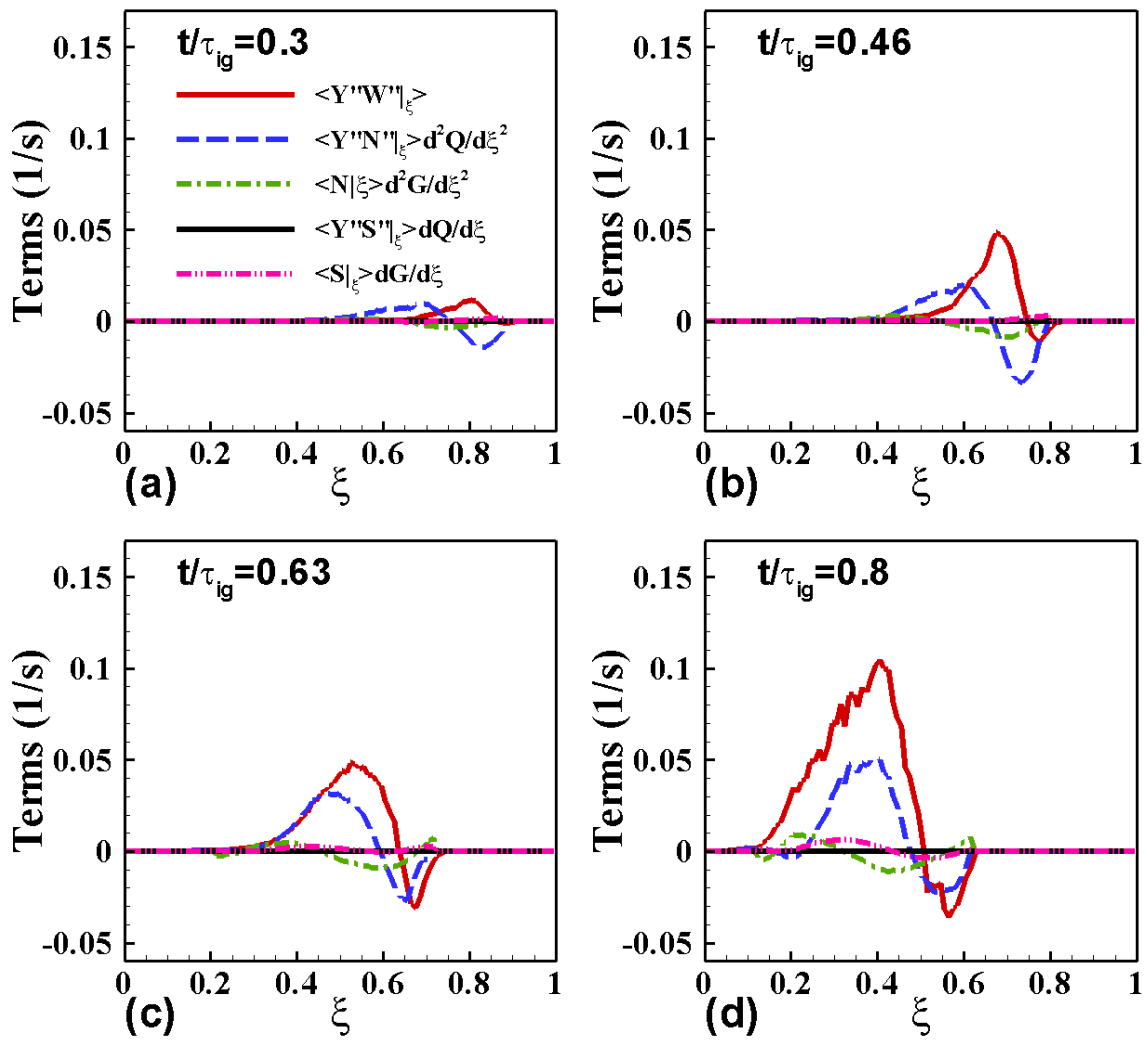

Fig. 15 Balance of terms in conditional variance equation, Eq. (8), for case octane-3 with $T^{\prime}=60 \mathrm{~K}$ at $t / \tau_{i g}=0.3,0.46,0.63$ and 0.8 .
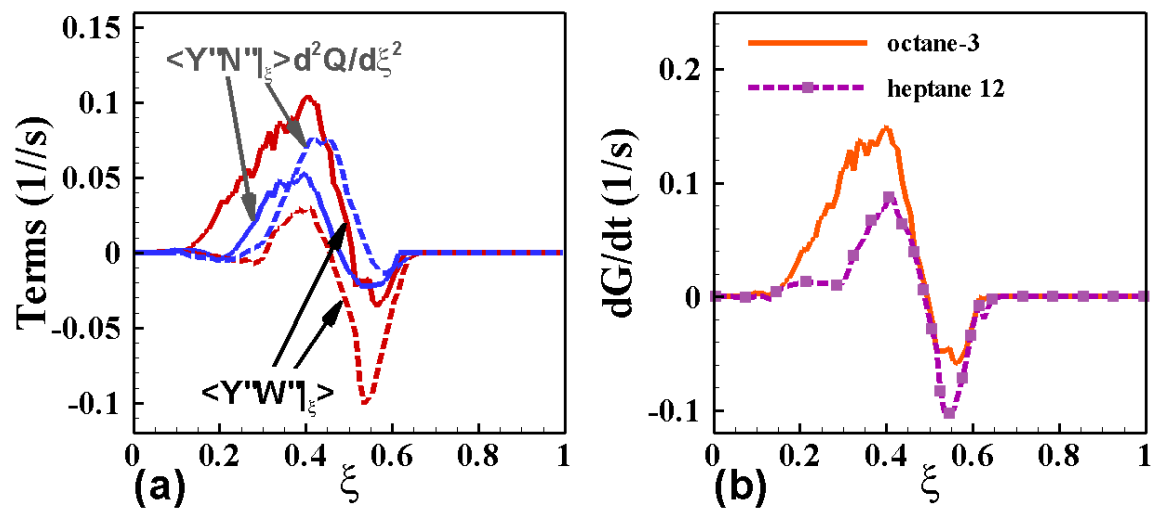

Fig. 16 Source terms a) and right side of conditional variance equation, Eq. (8), b) for cases octane- 3 and heptane- 12 with $T^{\prime}=60 \mathrm{~K}$ at $t / \tau_{i g}=0.8$ (Solid lines, case octane-3; dashed lines, case heptane-12). 